\title{
Criteria for generalized translation-invariant frames
}

\author{
Jakob Lemvig*, Jordy Timo van Velthoven ${ }^{\dagger}$
}

\begin{abstract}
This paper provides new sufficient and necessary conditions for the frame property of generalized translation-invariant systems. The conditions are formulated in the Fourier domain and consists of estimates involving the upper and lower frame bound. Contrary to known conditions of a similar nature, the estimates take the phase of the generating functions in consideration and not only their modulus. The possibility of phase cancellations makes these estimates optimal for tight frames. The results on generalized translation-invariant systems will be proved in the setting of locally compact abelian groups, but even for Euclidean space and the special case of (composite) wavelet systems the results are new.
\end{abstract}

\section{Introduction}

Deriving sufficient and necessary conditions for the frame property of structured function systems has a long history in time-frequency and time-scale analysis. In this paper we study a class of structured function systems known as generalized translation-invariant systems. These function systems offer a common framework for discrete and continuous structured function systems such as Gabor systems, composite wavelet systems and wave packet systems, but are also of independent interest.

\subsection{Overview and contributions}

The paper aims to derive necessary and sufficient conditions for the frame and Bessel property of generalized translation-invariant systems that are based on properties of the generating functions in the Fourier domain. The first results similar in nature go back to the very beginning of modern frame theory and the influential papers by Daubechies [19] and Daubechies, Grossmann and Meyer [21]. In [19], Daubechies provides general conditions on the generators and parameters of Gabor and wavelet systems to form a Bessel system or a frame for $L^{2}(\mathbb{R})$. These fundamental results attracted the attention of several groups of researchers [12, 15, 17, 27, 36, 45, 48, 51 and lead to improvements and generalizations over the subsequent decades, whose precise nature is discussed in Section 1.2 .

For the sake of clarity, in the remainder of this introduction, we focus on a subclass of discrete function systems, called generalized shift-invariant systems. In the setting of a locally compact abelian group $G$, written additively, a generalized shift-invariant system in $L^{2}(G)$ is

\footnotetext{
${ }^{*}$ Corresponding author. Technical University of Denmark, Department of Applied Mathematics and Computer Science, Matematiktorvet 303B, 2800 Kgs. Lyngby, Denmark, E-mail: jakle@dtu.dk

${ }^{\dagger}$ University of Vienna, Faculty of Mathematics, Oskar-Morgenstern-Platz 1, A-1090 Vienna, Austria, E-mail: jordy-timo.van-velthoven@univie.ac.at

2010 Mathematics Subject Classification. Primary 42C15, 42C40, Secondary: 43A32, 43A60, 43A70, 94 A12.

Key words and phrases. almost periodic functions, frame, frame bound, Gabor system, generalized translation-invariant system, necessary condition, sufficient condition, wavelet system
} 
a countable union of the form

$$
\bigcup_{j \in J}\left\{g_{j}(\cdot-\gamma): \gamma \in \Gamma_{j}\right\}
$$

for a collection of discrete, co-compact subgroups $\left\{\Gamma_{j}\right\}_{j \in J}$ in $G$ and a family of functions $\left\{g_{j}\right\}_{j \in J}$ in $L^{2}(G)$. A generalized shift-invariant system $\cup_{j \in J}\left\{g_{j}(\cdot-\gamma)\right\}_{\gamma \in \Gamma_{j}}$ in $L^{2}(G)$ is called a frame for $L^{2}(G)$ whenever there exist constants $A, B>0$, called frame bounds, such that

$$
A\|f\|_{2}^{2} \leq \sum_{j \in J} \sum_{\gamma \in \Gamma_{j}}\left|\left\langle f, g_{j}(\cdot-\gamma)\right\rangle\right|^{2} \leq B\|f\|_{2}^{2}
$$

for all $f \in L^{2}(G)$. A family $\cup_{j \in J}\left\{g_{j}(\cdot-\gamma)\right\}_{\gamma \in \Gamma_{j}}$ satisfying the upper frame bound is called a Bessel sequence and a frame for which the frame bounds can be chosen equal is called tight.

Frames in $L^{2}(G)$ are of interest in applications in, e.g., signal analysis and functional analysis, as they guarantee unconditionally $L^{2}$-convergent and stable expansions of functions in $L^{2}(G)$. Indeed, given a frame $\cup_{j \in J}\left\{g_{j}(\cdot-\gamma)\right\}_{\gamma \in \Gamma_{j}}$ in $L^{2}(G)$, there exists a system $\cup_{j \in J}\left\{\tilde{g}_{j, \gamma}\right\}_{\gamma \in \Gamma_{j}}$ such that every function $f \in L^{2}(G)$ possesses an expansion of the form

$$
f=\sum_{j \in J} \sum_{\gamma \in \Gamma_{j}}\left\langle f, \tilde{g}_{j, \gamma}\right\rangle g_{j}(\cdot-\gamma)=\sum_{j \in J} \sum_{\gamma \in \Gamma_{j}}\left\langle f, g_{j}(\cdot-\gamma)\right\rangle \tilde{g}_{j, \gamma}
$$

with unconditional norm convergence.

Verifying the frame inequalities (1.1) directly is often an impossible task. However, for many special cases simple sufficient and necessary conditions for the frame property are known. The new criteria presented in this paper will be derived under two unconditionally convergence properties (called $p$-UCP for $p=1, \infty$ ) that will be introduced in Definition 2.2. The $p$-UCP is a mild convergence property that guarantees almost periodicity of an auxiliary function $w_{f}$, essential to our analysis, that will be introduced in Section 2.2. The $\infty$-UCP will simply be assumed throughout the remainder of this subsection; here, for simplicity, we also assume that the Lebesgue Differentiation Theorem holds on $\widehat{G}$. The frame criteria are phrased as estimates involving the functions $t_{\alpha}: \widehat{G} \rightarrow \mathbb{C}, \alpha \in \bigcup_{j \in J} \Gamma_{j}^{\perp}$, defined, whenever convergent, as

$$
t_{\alpha}(\omega)=\sum_{j \in J: \alpha \in \Gamma_{j}^{+}} \frac{1}{\mathrm{~d}\left(\Gamma_{j}\right)} \hat{g}_{j}(\omega) \overline{\hat{g}_{j}(\omega+\alpha)}, \quad \text { a.e. } \omega \in \widehat{G}
$$

where $\Gamma_{j}^{\perp}$ is the dual lattice of $\Gamma_{j}$, and $\mathrm{d}\left(\Gamma_{j}\right)$ denotes the covolume of $\Gamma_{j}$.

The sufficient condition presented in Theorem 3.3 states that a system $\cup_{j \in J}\left\{g_{j}(\cdot-\gamma)\right\}_{\gamma \in \Gamma_{j}}$ forms a frame for $L^{2}(G)$ with bounds $A_{1}$ and $B_{1}$ if

$$
B_{1}:=\operatorname{esssup}_{\omega \in \widehat{G}} \sum_{\alpha \in \bigcup_{j \in J} \Gamma_{j}^{\perp}}\left|t_{\alpha}(\omega)\right|<\infty
$$

and

$$
A_{1}:=\operatorname{essinf}_{\omega \in \widehat{G}}\left(t_{0}(\omega)-\sum_{\alpha \in \bigcup_{j \in J} \Gamma_{j}^{\perp} \backslash\{0\}}\left|t_{\alpha}(\omega)\right|\right)>0 .
$$

The subscript $p$ is used in the constants $A_{p}$ and $B_{p}$ to indicate the relation with the $\ell_{p}$ norm of the sequence $\left\{t_{\alpha}(\omega)\right\}_{\alpha}$ for a.e. $\omega \in \widehat{G}$. Contrary to previously known sufficient conditions 
for generalized shift-invariant systems, the estimates (1.3) and (1.4) take the phase of the generating functions in consideration and not only their modulus. To be more precise, the previously known sufficient conditions are not based on the functions $t_{\alpha}: \widehat{G} \rightarrow \mathbb{C}$, but are based on the non-negative functions

$$
\widehat{G} \ni \omega \mapsto \sum_{j \in J: \alpha \in \Gamma_{j}^{+}} \frac{1}{\mathrm{~d}\left(\Gamma_{j}\right)}\left|\hat{g}_{j}(\omega) \hat{g}_{j}(\omega+\alpha)\right| \in[0, \infty[.
$$

By considering the phase of the generating functions, the estimates (1.3) and (1.4) allow, for each $\alpha \in \bigcup_{j \in J} \Gamma_{j}^{\perp}$, for phase cancellations in the sum over $\left\{j \in J: \alpha \in \Gamma_{j}^{\perp}\right\}$ and therefore often lead to improvements of the known estimates, see, e.g., Example 3.3 for an orthonormal basis demonstrating this. In fact, the estimates (1.3) and (1.4) are optimal for tight frames in the sense that they recover precisely the frame bound.

The obtained necessary condition (Theorem 3.2) asserts that if $\cup_{j \in J}\left\{g_{j}(\cdot-\gamma)\right\}_{\gamma \in \Gamma_{j}}$ is a Bessel sequence in $L^{2}(G)$ with upper frame bound $B$, then

$$
B \geq B_{2}:=\operatorname{ess}_{\omega \in \widehat{G}}\left(\sum_{\alpha \in \bigcup_{j \in J} \Gamma_{j}^{\perp}}\left|t_{\alpha}(\omega)\right|^{2}\right)^{1 / 2} .
$$

Combining this with the known fact that for a frame $\cup_{j \in J}\left\{g_{j}(\cdot-\gamma)\right\}_{\gamma \in \Gamma_{j}}$ for $L^{2}(G)$ with lower bound $A>0$ necessarily $A_{\infty}:=\operatorname{essinf}_{\omega \in \widehat{G}} t_{0}(\omega) \geq A$, it follows that $A \leq A_{\infty} \leq B_{2} \leq B$ is necessary for a frame $\cup_{j \in J}\left\{g_{j}(\cdot-\gamma)\right\}_{\gamma \in \Gamma_{j}}$ with bounds $A$ and $B$.

For the applicability of the frame expansions (1.2), it is not only essential to verify the frame inequalities (1.1), but also to provide good estimates of the frame bounds. The obtained necessary and sufficient conditions yield together frame bound estimates for generalized shiftinvariant systems. Indeed, for a Bessel system $\cup_{j \in J}\left\{g_{j}(\cdot-\gamma)\right\}_{\gamma \in \Gamma_{j}}$ with optimal upper bound $B>0$, the bound $B$ can be estimated by the snug bounds

$$
B_{2} \equiv \operatorname{esssup}_{\omega \in \widehat{G}}\left\|\left\{t_{\alpha}(\omega)\right\}_{\alpha}\right\|_{\ell^{2}} \leq B \leq \operatorname{ess}_{\omega \in \widehat{G}}\left\|\left\{t_{\alpha}(\omega)\right\}_{\alpha}\right\|_{\ell^{1}} \equiv B_{1} .
$$

If, furthermore, $\cup_{j \in J}\left\{g_{j}(\cdot-\gamma)\right\}_{\gamma \in \Gamma_{j}}$ is a frame in $L^{2}(G)$ with optimal lower bound $A>0$, then

$$
A_{1} \leq A \leq A_{\infty}
$$

The presented results hold not only for discrete frames and systems as discussed above, but also for their continuous and semi-continuous counterpart. To summarize, the main contributions of the paper are new necessary and sufficient conditions for the frame and Bessel property of generalized translation-invariant systems that are (i) derived under minimal assumptions, (ii) optimal for tight frames, (iii) verifiable and computable, and that provide (iv) snug frame bound estimates which collapse to equality for tight frames.

\subsection{Related work}

For shift-invariant systems, i.e., generalized shift-invariant systems with a single, fixed translation lattice $\Gamma$, the Bessel and frame properties can be characterized in terms of (bi-infinite) matrix-valued functions, known as dual Gramian matrices, as introduced by Ron and Shen [44], see also Janssen [35]. Consequently, the aforementioned necessary and sufficient conditions in 
(1.3), (1.4) and (1.5) can be derived 1 from simple norm estimates of bi-infinite Hermitian matrices $M=\left(m_{i, j}\right)_{i, j \in \Gamma^{\perp}}$ on $\ell^{2}\left(\Gamma^{\perp}\right)$, see [44, Section 1.6]. In particular, the estimates (1.6) and (1.7) are known for separable Gabor systems [34,48]; these estimates are the best-known improvement of Daubechies' Gabor frame bound estimates [19]. Furthermore, the dual Gramian characterization has, in a fiberization formulation [4, been extended to the setting of locally compact abelian groups [8,11,31]. Hence, for shift-invariant systems, or more generally, translation-invariant systems on such groups, the conditions (1.3), (1.4) and (1.5) follow from these characterizations and should not be considered new.

For function systems that are not shift-invariant, the fiberization characterization breaks down. In spite of this, Ron and Shen [49] obtained dual Gramian-type2 characterizations for special types of generalized shift-invariant systems in $L^{2}\left(\mathbb{R}^{d}\right)$. For example, for generalized shift-invariant systems satisfying the finite intersection (FI) condition (i.e., the intersection of any finite subfamily of the lattices $\left\{\Gamma_{j}\right\}_{j \in J}$ is a full-rank lattice), the Bessel property can be characterized by the norm of the dual Gramian matrices since the FI condition essentially reduces the analysis to standard dual Gramian analysis. On the other hand, many generalized shift-invariant systems violate the FI condition, e.g., systems with both rational and nonrational lattices. For lower frame bound characterizations by dual Gramian analysis additional restrictions on the lattices and generators are needed, most notably the so-called small tail condition. To handle wavelet systems associated with expansive, but not necessarily integer, matrix dilations, other assumptions on the family of lattices and generators are made such as the notions of temperateness and roundedness in generalized shift-invariant systems, cf. [49] for definitions. However, we stress that none of the used assumptions in [49] are weak enough to allow for dual Gramian characterization of wavelet frames associated with arbitrary real, expansive dilations.

An alternative route for deriving necessary and sufficient conditions for wavelet frames with integer, expansive dilations goes through quasi-affine systems [16, 46, 47]. This link is known to generalize to rational, expansive dilations [6], although one has to consider a family of quasiaffine systems to capture the frame property of the given wavelet system. Since quasi-affine systems are shift-invariant, sufficient and necessary conditions for rational wavelet systems are readily available. We stress that such estimates differ slightly from the ones presented in this paper. The estimates presented in 45 for wavelet systems with integer, expansive dilations utilize the quasi-affine route, but they ignore the phase of the wavelet generator and are therefore not optimal for tight frames.

The approach we follow relies on a connection between the frame properties of generalized shift-invariant systems and an associated almost periodic auxiliary function [28, 33, 41,51, Our methods are closely related to the work of Hernández, Labate and Weiss [28], but while [28] is concerned with tight frame characterization using uniqueness of the coefficients of almost periodic Fourier series, we focus on non-tight frames by bounding the Fourier series. The connection to Fourier analysis is valid under the 1-UCP, which is weak enough to provide sufficient and necessary conditions for wavelet frames in $L^{2}\left(\mathbb{R}^{d}\right)$ associated with any real, expansive dilation matrix and any translation lattice. The 1-UCP is even weak enough to handle every choice of real, invertible dilation (not necessarily expansive) almost surely with respect to the Haar measure on $\mathrm{GL}_{d}(\mathbb{R})$, see Section 4.2 .

To wrap up the discussion, no necessary or sufficient conditions, optimal for tight frames,

\footnotetext{
${ }^{1}$ For example, the necessary condition in (1.5) for shift-invariant systems follows from the norm estimate $\|M\| \geq\left(\sum_{j \in \Gamma^{\perp}}\left|m_{0, j}\right|^{2}\right)^{1 / 2}$ by noticing that the 0th column the dual Gramian matrix at $\omega \in \widehat{G}$ is $\left\{t_{\alpha}(\omega)\right\}_{\alpha \in \Gamma^{\perp}}$.

${ }^{2}$ For generalized shift-invariant systems the direct link using one dual Gramian matrix for each fiber has to be replaced by a less direct link of infinite families of finite matrices for each fiber.
} 
are currently known for wavelet systems associated with expansive, real dilations. In fact, the lack of optimal frame bound estimates for such systems lead Christensen [14] to ask whether sufficient conditions as in (1.3) and (1.4) can also be obtained for wavelet systems with noninteger dilations. The sufficient conditions obtained in this paper answer this question in the affirmative.

\subsection{Outline}

The paper is organized as follows. In Section 2 we introduce generalized translation-invariant systems and the 1-UCP condition in the setting of locally compact abelian groups. The main results on generalized translation-invariant systems are presented in Section 3 . Necessary and sufficient conditions for generalized translation-invariant frames are contained in Section 3.1 and 3.2. respectively. In Section 3.3 we compare the obtained frame bound estimates with known estimates. Section 4 is devoted to applications and examples. Gabor systems and wavelet systems are considered in Section 4.1 and Section 4.2, respectively. Finally, we consider composite wavelet and cone-adapted shearlet systems in Section 4.3, and we derive new frame characterizations of the continuous $\ell$-th order $\alpha$-shearlet transform in Section 4.4 .

\section{Generalized translation-invariant systems}

Throughout this paper, $G$ will denote a second countable locally compact abelian group. The character group of $G$ is denoted by $\widehat{G}$ and forms a second countable locally compact abelian group itself. Both $G$ and $\widehat{G}$ are $\sigma$-compact and metrizable. The group operation in both $G$ and $\widehat{G}$ is written additively as + and the identity element is denoted by 0 . The Haar measure on $G$ will be denoted by $\mu_{G}$. It is assumed that the Haar measure on $G$ is given and that the Haar measure on $\widehat{G}$ is the Plancherel measure. The subset $\Gamma \subseteq G$ will denote a closed, co-compact subgroup of $G$, i.e., the quotient space $G / \Gamma$ is compact. In this case, the annihilator $\Gamma^{\perp}$ of $\Gamma$ is the countable, discrete subgroup $\Gamma^{\perp}:=\{\omega \in \widehat{G} \mid \omega(x)=0, \forall x \in \Gamma\}$. It is assumed that the Haar measure on $\Gamma$ is given and that the Haar measure on $G / \Gamma$ is the unique quotient measure provided by Weil's integral formula. Using this quotient measure $\mu_{G / \Gamma}$ on $G / \Gamma$, the covolume or size of the subgroup $\Gamma \subseteq G$ is defined as $\mathrm{d}(\Gamma):=\mu_{G / \Gamma}(G / \Gamma)$.

\subsection{Generalized translation-invariant frames}

The function systems defined next form the central object of this paper. Here, the translate of a function $f \in L^{2}(G)$ by $y \in G$ is denoted as $T_{y} f:=f(\cdot-y)$.

Definition 2.1. Let $J$ be a countable index set. For each $j \in J$, let $\Gamma_{j} \subseteq G$ be a closed, co-compact subgroup, and let $P_{j}$ be an arbitrary (countable or uncountable) index set. For a given family of functions $\cup_{j \in J}\left\{g_{j, p}\right\}_{p \in P_{j}} \subset L^{2}(G)$, the collection of translates

$$
\bigcup_{j \in J}\left\{T_{\gamma} g_{j, p}\right\}_{\gamma \in \Gamma_{j}, p \in P_{j}}
$$

is called a generalized translation-invariant (GTI) system in $L^{2}(G)$.

We stress that the translation subgroups $\Gamma_{j} \subset G, j \in J$, are not assumed to be discrete, but merely closed and co-compact. In $G=\mathbb{R}^{d}$ (and $\widehat{G}=\mathbb{R}^{d}$ ), each closed, co-compact subgroup $\Gamma_{j}$ is of the form $C\left(\mathbb{Z}^{k} \times \mathbb{R}^{d-k}\right)$ for some $0 \leq k \leq d$ and $C \in \mathrm{GL}_{d}(\mathbb{R})$. The (discrete) annihilator $\Gamma_{j}^{\perp}$ is then given by $\left(C^{T}\right)^{-1}\left(\mathbb{Z}^{k} \times\{0\}^{d-k}\right)$.

Following [32], it is assumed that the generating functions of a generalized translation-invariant systems satisfy the following three standing hypotheses. For each $j \in J$ : 
(I) The triple $\left(P_{j}, \Sigma_{P_{j}}, \mu_{P_{j}}\right)$ forms a $\sigma$-finite measure space;

(II) The mapping $p \mapsto g_{j, p}$ from $\left(P_{j}, \Sigma_{P_{j}}\right)$ into $\left(L^{2}(G), \mathcal{B}_{L^{2}(G)}\right)$ is $\Sigma_{P_{j}}$-measurable, where $\mathcal{B}_{L^{2}(G)}$ denotes the Borel $\sigma$-algebra on $L^{2}(G)$;

(III) The mapping $(p, x) \mapsto g_{j, p}(x)$ from $\left(P_{j} \times G, \Sigma_{P_{j}} \otimes \mathcal{B}_{G}\right)$ into $\left(\mathbb{C}, \mathcal{B}_{\mathbb{C}}\right)$ is $\left(\Sigma_{P_{j}} \otimes \mathcal{B}_{G}\right)$ measurable, where $\mathcal{B}_{G}$ denotes the Borel $\sigma$-algebra on $G$;

For most applications in this paper, it suffices to take $\left\{P_{j}\right\}_{j \in J}$ to be countable index sets equipped with the counting measure, cf. Section 4 . In this case, the three standing hypotheses (II) -(III) are automatically satisfied.

A generalized translation-invariant system $\cup_{j \in J}\left\{T_{\gamma} g_{j, p}\right\}_{\gamma \in \Gamma_{j}, p \in P_{j}}$ is called a generalized translation-invariant frame for $L^{2}(G)$, with respect to $\left\{L^{2}\left(P_{j} \times \Gamma_{j}\right) \mid j \in J\right\}$, whenever there exist two constants $A, B>0$, called the frame bounds, such that

$$
A\|f\|^{2} \leq \sum_{j \in J} \int_{P_{j}} \int_{\Gamma_{j}}\left|\left\langle f, T_{\gamma} g_{j, p}\right\rangle\right|^{2} d \mu_{\Gamma_{j}}(\gamma) d \mu_{P_{j}}(p) \leq B\|f\|^{2}
$$

for all $f \in L^{2}(G)$. A generalized translation-invariant system $\cup_{j \in J}\left\{T_{\gamma} g_{j, p}\right\}_{\gamma \in \Gamma_{j}, p \in P_{j}}$ satisfying the upper frame bound is called a Bessel system or a Bessel family in $L^{2}(G)$. For such a Bessel system, the frame operator $S: L^{2}(G) \rightarrow L^{2}(G)$, associated with $\cup_{j \in J}\left\{T_{\gamma} g_{j, p}\right\}_{\gamma \in \Gamma_{j}, p \in P_{j}}$, is defined weakly by equating $\langle S f, f\rangle$ with the central term of (2.1).

In order to check whether a generalized translation-invariant system forms a Bessel system or a frame for $L^{2}(G)$, it suffices to check the frame condition on a dense subspace of $L^{2}(G)$. Let $\mathcal{E}$ denote the set of all closed Borel sets $E \subseteq \widehat{G}$ satisfying $\mu_{\widehat{G}}(E)=0$. For a fixed $E \in \mathcal{E}$, define the dense subspace $\mathcal{D}_{E}(G)$ of $L^{2}(G)$ as

$$
\mathcal{D}_{E}(G)=\left\{f \in L^{2}(G) \mid \hat{f} \in L^{\infty}(\widehat{G}) \text { and } \exists K \subset \widehat{G} \backslash E \text { compact with } \hat{f} \mathbb{1}_{K}=\hat{f} \text { a.e. }\right\},
$$

where $\hat{f}$ denotes the Fourier transform of $f \in L^{2}(G)$ and $\mathbb{1}_{K}$ the characteristic function on $K$. A function $f \in \mathcal{D}_{E}(G)$ has the property that $\hat{f}$ is zero almost everywhere on $E+B(0, \delta)$ for some $\delta>0$, where $B(0, \delta)$ denotes the open ball of radius $\delta$, since supp $\hat{f}$ is compact, $E$ is closed, and $\operatorname{supp} \hat{f} \cap E=\emptyset$.

We consider the set $E \in \mathcal{E}$, called the blind spot, as fixed, but arbitrary. The actual choice of $E$ depends on the considered application. For wavelet systems, the blind spot $E$ is usually chosen to be the complement $\mathcal{O}^{c}$ of the dual orbit $\mathcal{O} \subseteq \widehat{G}$ of the dilation group [23], whereas for Gabor systems it usually suffices to take $E=\emptyset$.

\subsection{Unconditional convergence property and Fourier analysis}

This section is devoted to the 1-UCP condition and to generalized Fourier series of almost periodic functions essential to our study of generalized translation-invariant frames. Recall that an almost periodic function is the uniform limit of trigonometric polynomials. The space of almost periodic functions is denoted by $\operatorname{AP}(G)$.

Given a generalized translation-invariant system $\cup_{j \in J}\left\{T_{\gamma} g_{j, p}\right\}_{\gamma \in \Gamma_{j}, p \in P_{j}}$ and a function $f \in$ $\mathcal{D}_{E}(G)$, define, for $j \in J$, the map $w_{f, j}: G \rightarrow[0, \infty]$ by

$$
w_{f, j}(x)=\int_{P_{j}} \int_{\Gamma_{j}}\left|\left\langle T_{x} f, T_{\gamma} g_{j, p}\right\rangle\right|^{2} d \mu_{\Gamma_{j}}(\gamma) d \mu_{P_{j}}(p) .
$$


By the standing hypotheses (II)-(III), the integrals in (2.2) are well-defined. Throughout this section, we will further assume that

$$
\int_{P_{j}}\left|\hat{g}_{j, p}(\cdot)\right|^{2} d \mu_{P_{j}}(p) \in L_{\mathrm{loc}}^{1}(\widehat{G}) .
$$

It can be shown, using arguments from [32,38, that the integrability condition (2.3) ensures that each $w_{f, j}$ is a trigonometric polynomial, in particular, that $w_{f, j}$ are $\Gamma_{j}$-periodic, continuous, and bounded.

Next, we define $w_{f}: G \rightarrow[0, \infty]$ as the following sum of non-negative, trigonometric polynomials

$$
w_{f}(x)=\sum_{j \in J} w_{f, j}(x)=\sum_{j \in J} \int_{P_{j}} \int_{\Gamma_{j}}\left|\left\langle T_{x} f, T_{\gamma} g_{j, p}\right\rangle\right|^{2} d \mu_{\Gamma_{j}}(\gamma) d \mu_{P_{j}}(p) .
$$

The function $w_{f}: G \rightarrow[0, \infty]$ is well-defined, but it might attain the value of positive infinity without any further assumptions on the generalized translation-invariant system.

Under a suitable regularity condition on the system $\cup_{j \in J}\left\{T_{\gamma} g_{j, p}\right\}_{\gamma \in \Gamma_{j}, p \in P_{j}}$, the function $w_{f}$ becomes almost periodic. The connection between the almost periodicity of $w_{f}$ and generalized translation-invariant frames was first used by Laugesen [40, 41, for wavelet systems and extended to arbitrary generalized shift-invariant (GSI) systems in $L^{2}\left(\mathbb{R}^{d}\right)$ by Hernández, Labate and Weiss [28].

The following regularity condition, sufficiently weak for our purposes, was introduced in 24.

Definition 2.2. Let $\cup_{j \in J}\left\{T_{\gamma} g_{j, p}\right\}_{\gamma \in \Gamma_{j}, p \in P_{j}}$ be a generalized translation-invariant system.

(i) The system $\cup_{j \in J}\left\{T_{\gamma} g_{j, p}\right\}_{\gamma \in \Gamma_{j}, p \in P_{j}}$ is said to satisfy the 1-unconditional convergence property (1-UCP), with respect to $E \in \mathcal{E}$, whenever, for all $f \in \mathcal{D}_{E}(G)$, the function $w_{f}: G \rightarrow \mathbb{C}$ is almost periodic and the series

$$
w_{f}=\sum_{j \in J} w_{f, j}
$$

converges unconditionally with respect to the mean $M: \operatorname{AP}(G) \rightarrow[0, \infty)$,

$$
M(|f|)=\lim _{n \rightarrow \infty} \frac{1}{\mu_{G}\left(H_{n}\right)} \int_{H_{n}}|f(x)| d \mu_{G}(x),
$$

where $\left(H_{n}\right)_{n \in \mathbb{N}}$ is any increasing sequence of open, relatively compact subsets $H_{n} \subseteq G$ with $G=\bigcup_{n \in \mathbb{N}} H_{n}$ and such that

$$
\lim _{n \rightarrow \infty} \frac{\mu_{G}\left(\left(x+H_{n}\right) \cap\left(G \backslash H_{n}\right)\right)}{\mu_{G}\left(H_{n}\right)}=0
$$

for all $x \in G$.

(ii) In case (2.4) holds with uniform convergence, the system $\cup_{j \in J}\left\{T_{\gamma} g_{j, p}\right\}_{\gamma \in \Gamma_{j}, p \in P_{j}}$ is said to satisfy the $\infty-U C P$ with respect to $E \in \mathcal{E}$. 
The 1-UCP is the weakest known assumption under which Fourier analysis of $w_{f}$ can be exploited to study the frame properties of the associated system. Note that almost periodicity of $w_{f}$ is assumed in 1-UCP, while, in $\infty$-UCP, it follows from the uniform convergence of (2.4).

Both 1-UCP and $\infty$-UCP are automatically satisfied whenever the generalized translation-invariant system $\cup_{j \in J}\left\{T_{\gamma} g_{j, p}\right\}_{\gamma \in \Gamma_{j}, p \in P_{j}}$ satisfies the local integrability condition (LIC) or the weaker $\alpha$-local integrability condition ( $\alpha$-LIC) introduced in [28] and [32, respectively. A system $\cup_{j \in J}\left\{T_{\gamma} g_{j, p}\right\}_{\gamma \in \Gamma_{j}, p \in P_{j}}$ is said to satisfy the $\alpha-L I C$, with respect to $E$, if

$$
\sum_{j \in J} \frac{1}{\mathrm{~d}\left(\Gamma_{j}\right)} \int_{P_{j}} \sum_{\alpha \in \Gamma_{j}^{\perp}} \int_{\widehat{G}}\left|\hat{f}(\omega) \hat{f}(\omega+\alpha) \hat{g}_{j, p}(\omega+\alpha) \hat{g}_{j, p}(\omega)\right| d \mu_{\widehat{G}}(\omega) d \mu_{P_{j}}(p)<\infty
$$

for all $f \in \mathcal{D}_{E}(G)$.

The generalized Fourier series of $w_{f}$ is stated in the next result, adapted from [24].

Proposition 2.3. Suppose $\cup_{j \in J}\left\{T_{\gamma} g_{j, p}\right\}_{\gamma \in \Gamma_{j}, p \in P_{j}}$ satisfies the 1 - $U C P$ with respect to $E \in \mathcal{E}$. Moreover, suppose that

$$
\sum_{j \in J} \frac{1}{\mathrm{~d}\left(\Gamma_{j}\right)} \int_{P_{j}}\left|\hat{g}_{j, p}(\cdot)\right|^{2} d \mu_{P_{j}}(p) \in L_{\mathrm{loc}}^{1}(\widehat{G} \backslash E)
$$

Then, for all $f \in \mathcal{D}_{E}(G)$, the Fourier-Bohr transform of $w_{f}: G \rightarrow \mathbb{C}$ at $\alpha \in \widehat{G}$ is

$$
\widehat{w_{f}}(\alpha):=M\left(w_{f} \cdot \bar{\alpha}\right)=\sum_{j \in J} \widehat{w_{f, j}}(\alpha)
$$

with absolute convergence. The generalized Fourier series of $w_{f}: G \rightarrow \mathbb{C}$ is given by

$$
w_{f}=\sum_{\alpha \in \bigcup_{j \in J} \Gamma_{j}^{\perp}} c_{\alpha} \alpha
$$

where the Fourier coefficients are given by

$$
c_{\alpha}=\int_{\widehat{G}} \hat{f}(\omega) \overline{\hat{f}(\omega+\alpha)} \sum_{j \in J: \alpha \in \Gamma_{j}^{+}} \frac{1}{\mathrm{~d}\left(\Gamma_{j}\right)} \int_{P_{j}} \overline{\hat{g}_{j, p}(\omega)} \hat{g}_{j, p}(\omega+\alpha) d \mu_{P_{j}}(p) d \mu_{\widehat{G}}(\omega) .
$$

Furthermore, if the $\infty$-UCP holds, then $w_{f}$ agrees pointwise with its generalized Fourier series (2.6).

Proof. The result, except for the specific form of the Fourier coefficients, can be found in [24]. Indeed, by [24, Proposition 3.10], the generalized Fourier series of $w_{f}: G \rightarrow \mathbb{C}$ is $w_{f}=\sum_{\alpha \in \bigcup_{j \in J} \Gamma_{j}^{\perp}} c_{\alpha} \alpha$ with coefficients

$$
c_{\alpha}=\sum_{j \in J: \alpha \in \Gamma_{j}^{\perp}} \int_{\widehat{G}} \hat{f}(\omega) \overline{\hat{f}(\omega+\alpha)} \frac{1}{\mathrm{~d}\left(\Gamma_{j}\right)} \int_{P_{j}} \overline{\hat{g}_{j, p}(\omega)} \hat{g}_{j, p}(\omega+\alpha) d \mu_{P_{j}}(p) d \mu_{\widehat{G}}(\omega) .
$$

For fixed $\alpha \in \bigcup_{j \in J} \Gamma_{j}^{\perp}$, the assumption (2.5), together with Cauchy-Schwarz' inequality, yields that

$$
\sum_{j \in J: \alpha \in \Gamma_{j}^{\perp}} \frac{1}{\mathrm{~d}\left(\Gamma_{j}\right)} \int_{P_{j}}\left|\hat{g}_{j, p}(\cdot) \hat{g}_{j, p}(\cdot+\alpha)\right| d \mu_{P_{j}}(p) \in L_{\mathrm{loc}}^{1}(\widehat{G} \backslash E),
$$


which in turn implies that

$$
\sum_{j \in J: \alpha \in \Gamma_{j}^{+}} \int_{\widehat{G}}\left|\hat{f}(\omega) \hat{f}(\omega+\alpha) \frac{1}{\mathrm{~d}\left(\Gamma_{j}\right)} \int_{P_{j}} \hat{g}_{j, p}(\omega) \hat{g}_{j, p}(\omega+\alpha) d \mu_{P_{j}}(p)\right| d \mu_{\widehat{G}}(\omega)<\infty .
$$

Thus, by Fubini-Tonelli's theorem, the series and integral defining $c_{\alpha}$ can be interchanged to obtain the desired form (2.7).

To ease notation, we define the following functions appearing implicitly in the Fourier coefficients in Proposition 2.3 .

Definition 2.4. Let $\cup_{j \in J}\left\{T_{\gamma} g_{j, p}\right\}_{\gamma \in \Gamma_{j}, p \in P_{j}}$ be a generalized translation-invariant system satisfying

$$
\sum_{j \in J} \frac{1}{\mathrm{~d}\left(\Gamma_{j}\right)} \int_{P_{j}}\left|\hat{g}_{j, p}(\omega)\right|^{2} d \mu_{P_{j}}(p)<\infty
$$

for $\mu_{\widehat{G}^{-}}$a.e. $\omega \in \widehat{G}$. For the system $\cup_{j \in J}\left\{T_{\gamma} g_{j, p}\right\}_{\gamma \in \Gamma_{j}, p \in P_{j}}$, the associated auto-correlation functions $t_{\alpha}, \alpha \in \cup_{j \in J} \Gamma_{j}^{\perp}$ are $\mu_{\widehat{G}^{-}}$a.e. defined by

$$
t_{\alpha}: \widehat{G} \rightarrow \mathbb{C}, \omega \mapsto \sum_{j \in J: \alpha \in \Gamma_{j}^{+}} \frac{1}{\mathrm{~d}\left(\Gamma_{j}\right)} \int_{P_{j}} \hat{g}_{j, p}(\omega) \overline{\hat{g}_{j, p}(\omega+\alpha)} d \mu_{P_{j}}(p) .
$$

Phrased in terms of the auto-correlation functions, the assumptions (2.5) and (2.8) require $t_{0}: \widehat{G} \rightarrow \mathbb{C}$ to be locally integrable and uniformly bounded, respectively. Any generalized translation-invariant system forming a Bessel family with upper bound $B>0$ satisfies

$$
t_{0}(\omega) \leq B
$$

for $\mu_{\widehat{G}^{-}}$a.e. $\omega \in \widehat{G}$, as shown in $28,32,38$. The assumptions of any of our results in Section 3 imply (2.8), hence the auto-correlation functions $t_{\alpha}$ will always be well-defined. In particular, assumption (2.3) is satisfied.

\section{Sufficient and necessary conditions for the frame property}

This section contains the main results of the paper. Section 3.1provides a necessary condition for the Bessel and frame property of a generalized translation-invariant system. Sufficient conditions for the Bessel and frame properties of generalized translation-invariant systems are presented in Section 3.2. The obtained sufficient conditions are compared to known frame bound estimates in Section 3.3

\subsection{Necessary conditions}

Inequality (2.9) is a necessary condition for the Bessel property of a generalized translationinvariant system. Under a weak regularity assumption, Theorem 3.2 below presents a much stronger necessary condition for a generalized translation-invariant system to form a Bessel family.

The proof of Theorem 3.2 makes use of a differentiation process for integrals on locally compact groups as in [22, Section 2]. In order to apply this, the following notion is useful, cf. [22, Definition 2.1]. 
Definition 3.1. Let $G$ be a locally compact group with Haar measure $\mu_{G}$. A decreasing sequence $\left(U_{k}\right)_{k \in \mathbb{N}}$ of finite measure Borel sets is called a $D^{\prime}$-sequence in $G$ if every neighborhood of 0 contains some $U_{k}$, and if there exists a constant $C>0$ such that

$$
0<\mu_{G}\left(U_{k}-U_{k}\right) \leq C \mu_{G}\left(U_{k}\right)
$$

for all $k \in \mathbb{N}$, where $U_{k}-U_{k}:=\left\{u-v: u, v \in U_{k}\right\}$.

Given a $D^{\prime}$-sequence $\left(U_{k}\right)_{k \in \mathbb{N}}$ for $G$ and an $f \in L_{\text {loc }}^{1}(G)$, a point $x_{0} \in G$ satisfying

$$
\lim _{k \rightarrow \infty} \frac{1}{\mu_{G}\left(U_{k}\right)} \int_{x_{0}+U_{k}} f(x) d \mu_{G}(x)=f\left(x_{0}\right)
$$

is called a Lebesgue point of $f$. Lebesgue's differentiation theorem [29, Theorem 44.18] asserts that the set of Lebesgue points of any $f \in L_{\text {loc }}^{1}(G)$ has full measure, or equivalently, that (3.1) holds for a.e. $x_{0} \in G$.

In [8], it is shown that any compactly generated abelian Lie group $G$ admits a $D^{\prime}$-sequence. As a consequence, any locally compact abelian group $G$ of the form $G=\mathbb{R}^{d} \times \mathbb{T}^{m} \times \mathbb{Z}^{n} \times F$, where $d, m, n \in \mathbb{N}$ and $F$ is finite, possesses a $D^{\prime}$-sequence. On the other hand, Bownik and Ross [8] also show that some infinite dimensional locally compact abelian groups, e.g., the tubby torus $\mathbb{T}^{\aleph_{0}}$, do not admit a $D^{\prime}$-sequence.

Theorem 3.2. Let $G$ be a locally compact abelian group such that $\widehat{G}$ admits a $D^{\prime}$-sequence. Let $\cup_{j \in J}\left\{T_{\gamma} g_{j, p}\right\}_{\gamma \in \Gamma_{j}, p \in P_{j}}$ be a generalized translation-invariant system satisfying the 1-UCP such that the Fourier series of $w_{f}$ converges unconditionally pointwise to $w_{f}\left(x_{0}\right)$ for some $x_{0} \in G$. Suppose $\cup_{j \in J}\left\{T_{\gamma} g_{j, p}\right\}_{\gamma \in \Gamma_{j}, p \in P_{j}}$ forms a Bessel system in $L^{2}(G)$ with Bessel bound $B$. Then

$$
B \geq B_{2}:=\operatorname{esssup}_{\omega \in \widehat{G}}\left(\sum_{\alpha \in \bigcup_{j \in J} \Gamma_{\frac{1}{j}}^{\perp}}\left|t_{\alpha}(\omega)\right|^{2}\right)^{1 / 2} .
$$

Moreover, if $\cup_{j \in J}\left\{T_{\gamma} g_{j, p}\right\}_{\gamma \in \Gamma_{j}, p \in P_{j}}$, in addition, forms a frame with lower frame bound $A>0$, then $A_{\infty}:=\operatorname{essinf}_{\omega \in \widehat{G}} t_{0}(\omega) \geq A$.

Proof. The "moreover"-part is a consequence of [24, Theorem 3.13]. The remainder of the proof is divided into three steps:

Step 1: Rewriting the frame operator. Let $S: L^{2}(G) \rightarrow L^{2}(G)$ denote the frame operator associated with the Bessel family $\cup_{j \in J}\left\{T_{\gamma} g_{j, p}\right\}_{\gamma \in \Gamma_{j}, p \in P_{j}}$. The map $\left(f_{1}, f_{2}\right) \mapsto\left\langle S f_{1}, f_{2}\right\rangle$ is a well-defined, bounded sesquilinear form on $L^{2}(G) \times L^{2}(G)$ with

$$
\left|\left\langle S f_{1}, f_{2}\right\rangle\right| \leq B\left\|f_{1}\right\|_{2}\left\|f_{2}\right\|_{2}
$$

for all $f_{1}, f_{2} \in L^{2}(G)$. The frame operator is related to the function $w_{f}: G \rightarrow \mathbb{C}$ introduced in (2.4) by $w_{f}(x)=\left\langle S T_{x} f, T_{x} f\right\rangle$, in particular, $w_{f}(0)=\langle S f, f\rangle$ for $f \in \mathcal{D}_{E}(G)$. By translation invariance of $\mathcal{D}_{E}(G)$, we can assume $x_{0}=0$, that is, pointwise convergence of the Fourier series of $w_{f}$ at the origin to $w_{f}(0)$. By Proposition 2.3, it then follows that

$$
\langle S f, f\rangle=\sum_{\alpha \in \bigcup_{j \in J} \Gamma_{j}^{\perp}} \int_{\widehat{G}} \hat{f}(\omega) \overline{\hat{f}(\omega+\alpha)} \overline{t_{\alpha}(\omega)} d \mu_{\widehat{G}}(\omega)
$$


for all $f \in \mathcal{D}_{E}(G)$. Here, each auto-correlation function $t_{\alpha}: \widehat{G} \rightarrow \mathbb{C}$ is well-defined with $\left\|t_{\alpha}\right\|_{\infty} \leq B$ by (2.9). The identity (3.4), together with an application of the polarization identity for sesquilinear forms and the bound (3.3), therefore gives

$$
\left|\sum_{\alpha \in \cup_{j \in J} \Gamma_{j}^{\perp}} \int_{\widehat{G}} \hat{f}_{1}(\omega) \overline{\hat{f}_{2}(\omega+\alpha)} \overline{t_{\alpha}(\omega)} d \mu_{\widehat{G}}(\omega)\right| \leq B\left\|f_{1}\right\|_{2}\left\|f_{2}\right\|_{2}
$$

for all $f_{1}, f_{2} \in \mathcal{D}_{E}(G)$. For clarity, we define $c_{\alpha}:=\int_{\widehat{G}} \hat{f}_{1}(\omega) \overline{\hat{f}_{2}(\omega+\alpha)} \overline{t_{\alpha}(\omega)} d \mu_{\widehat{G}}(\omega)$ for $\alpha \in$ $\Lambda:=\bigcup_{j \in J} \Gamma_{j}^{\perp}$. Then (3.5) simply reads $\left|\sum_{\alpha} c_{\alpha}\right| \leq B\left\|f_{1}\right\|_{2}\left\|f_{2}\right\|_{2}$.

Step 2: Construction of test functions $f_{1}, f_{2}$. First, we assume that $E=\emptyset$ in the 1-UCP assumption. Let $\omega_{0} \in \widehat{G}$ be a common Lebesgue point of $\left|t_{\alpha}\right|^{2} \in L^{\infty}(\widehat{G}) \subset L_{\text {loc }}^{1}(\widehat{G})$ for all $\alpha \in \Lambda$. The dual group $\widehat{G}$ is second countable, whence metrizable. The metric $d_{\widehat{G}}$ inducing the given topology on $\widehat{G}$ can be chosen to be translation-invariant. Let $\sigma: \mathbb{N} \rightarrow J$ be a bijection and define $\Lambda_{m, n}:=\bigcup_{i=1}^{n} \Gamma_{\sigma(i)}^{\perp} \cap B(0, m)$, where $B(0, m)$ denotes the open ball, relative to $d_{\widehat{G}}$, with radius $m>0$ and center $0 \in \widehat{G}$. Then, given any $\alpha \in \Lambda$, there exists $m, n \in \mathbb{N}$ such that $\alpha \in \Lambda_{m, n}$.

For fixed $m, n \in \mathbb{N}$, set $\delta_{m, n}:=\min \left\{d_{\widehat{G}}\left(\alpha, \alpha^{\prime}\right): \alpha, \alpha^{\prime}, \in \Lambda_{m, n}\right.$ with $\left.\alpha \neq \alpha^{\prime}\right\}$. Note that $\delta_{m, n}>0$ since $\Lambda_{m, n}$ is a finite set. Let $\left(U_{k}\right)_{k \in \mathbb{N}}$ be a $D^{\prime}$-sequence in $\widehat{G}$. The sets $U_{k}$ lie eventually inside an arbitrary neighborhood of $0 \in \widehat{G}$. Thus, by local compactness of $\widehat{G}$, we can assume without loss of generality that $U_{1}$ is relatively compact. Moreover, we let $K \in \mathbb{N}$ be so that $U_{k} \subset B\left(0, \delta_{m, n} / 2\right)$ for all $k \geq K$. Then, for all $k \geq K$,

$$
\mu_{\widehat{G}}\left(\left(\alpha+U_{k}\right) \cap\left(\alpha^{\prime}+U_{k}\right)\right)=0 \quad \text { for all } \alpha, \alpha^{\prime} \in \Lambda_{m, n} \text { with } \alpha \neq \alpha^{\prime} .
$$

Define $f_{1} \in \mathcal{D}_{\emptyset}(G)$ by $\hat{f}_{1}:=\mu_{\widehat{G}}\left(U_{k}\right)^{-1 / 2} \mathbb{1}_{\omega_{0}+U_{k}}$. For $k \geq K$, define $h: \widehat{G} \rightarrow \mathbb{C}$ on $\omega_{0}+U_{k}+\Lambda_{m, n}$ by

$$
h(\omega+\alpha)=\overline{t_{\alpha}(\omega)} \quad \text { for a.e. } \omega \in \omega_{0}+U_{k}
$$

for each $\alpha \in \Lambda_{m, n}$ and by $h(\omega)=0$ for $\omega \in \widehat{G} \backslash\left(\omega_{0}+U_{k}+\Lambda_{m, n}\right)$. The property (3.6) of $U_{k}$ guarantees that $h$ is well-defined. Let $\hat{f}_{2}:=\|h\|_{2}^{-1} h$. Then $f_{2} \in \mathcal{D}_{\emptyset}(G)$ with $\left\|f_{2}\right\|_{2}=1$. A direct calculation entails

$$
\begin{aligned}
\sum_{\alpha \in \Lambda_{m, n}} c_{\alpha} & =\sum_{\alpha \in \Lambda_{m, n}} \int_{\widehat{G}} \hat{f}_{1}(\omega) \overline{\hat{f}_{2}(\omega+\alpha)} \overline{t_{\alpha}(\omega)} d \mu_{\widehat{G}}(\omega) \\
& =\|h\|_{2}^{-1} \sum_{\alpha \in \Lambda_{m, n}} \frac{1}{\mu_{\widehat{G}}\left(U_{k}\right)^{1 / 2}} \int_{\omega_{0}+U_{k}}\left|t_{\alpha}(\omega)\right|^{2} d \mu_{\widehat{G}}(\omega) \\
& =\frac{1}{\mu_{\widehat{G}}\left(U_{k}\right)^{1 / 2}}\left(\sum_{\alpha \in \Lambda_{m, n}} \int_{\omega_{0}+U_{k}}\left|t_{\alpha}(\omega)\right|^{2} d \mu_{\widehat{G}}(\omega)\right)^{-1 / 2}\left(\sum_{\alpha \in \Lambda_{m, n}} \int_{\omega_{0}+U_{k}}\left|t_{\alpha}(\omega)\right|^{2} d \mu_{\widehat{G}}(\omega)\right) \\
& =\left(\sum_{\alpha \in \Lambda_{m, n}} \frac{1}{\mu_{\widehat{G}}\left(U_{k}\right)} \int_{\omega_{0}+U_{k}}\left|t_{\alpha}(\omega)\right|^{2} d \mu_{\widehat{G}}(\omega)\right)^{1 / 2}
\end{aligned}
$$

for any $m, n \in \mathbb{N}$ and $k \geq K$. An application of Lebesgue's differentiation theorem [29, Theorem 44.18] next gives

$$
\lim _{k \rightarrow \infty}\left(\sum_{\alpha \in \Lambda_{m, n}} \frac{1}{\mu_{\widehat{G}}\left(U_{k}\right)} \int_{\omega_{0}+U_{k}}\left|t_{\alpha}(\omega)\right|^{2} d \mu_{\widehat{G}}(\omega)\right)^{1 / 2}=\left(\sum_{\alpha \in \Lambda_{m, n}}\left|t_{\alpha}\left(w_{0}\right)\right|^{2}\right)^{1 / 2}
$$


for any $m, n \in \mathbb{N}$.

Step 3: An $\varepsilon$-argument. For arbitrary $\varepsilon>0$, there exist $M, N \in \mathbb{N}$ such that, for all $m \geq M, n \geq N$,

$$
\left|\sum_{\alpha \in \Lambda} c_{\alpha}-\sum_{\alpha \in \Lambda_{m, n}} c_{\alpha}\right| \leq \varepsilon .
$$

Using (3.5) and (3.7), it now follows by the triangle inequality that

$$
\left(\sum_{\alpha \in \Lambda_{m, n}}\left|t_{\alpha}\left(w_{0}\right)\right|^{2}\right)^{1 / 2} \leq B+\varepsilon
$$

for $m \geq M$ and $n \geq N$. Since $\sum_{\alpha \in \Lambda_{m, n}}\left|t_{\alpha}\left(w_{0}\right)\right|^{2}$ is a bounded and monotone increasing sequence of $n$ and $m$, the limit

$$
\left(\sum_{\alpha \in \Lambda}\left|t_{\alpha}\left(w_{0}\right)\right|^{2}\right)^{1 / 2}=\sup _{m \geq M, n \geq N}\left(\sum_{\alpha \in \Lambda_{m, n}}\left|t_{\alpha}\left(w_{0}\right)\right|^{2}\right)^{1 / 2}
$$

exists.

Since $\varepsilon>0$ was taken arbitrary, it follows that

$$
\left(\sum_{\alpha \in \Lambda}\left|t_{\alpha}\left(\omega_{0}\right)\right|^{2}\right)^{1 / 2} \leq B
$$

Being a countable union of null sets, the complement of the set of common Lebesgue points of $\left|t_{\alpha}\right|^{2}, \alpha \in \Lambda$, is a null set. We conclude that (3.8) holds for $\mu_{\widehat{G}^{-}}$a.e. $\omega_{0} \in \widehat{G}$, which completes the proof for the case $E=\emptyset$.

Lastly, consider the case that $\cup_{j \in J}\left\{T_{\gamma} g_{j, p}\right\}_{\gamma \in \Gamma_{j}, p \in P_{j}}$ satisfies the 1-UCP with respect to an arbitrary $E \in \mathcal{E}$. In this case the Fourier supports of $f_{1}$ and $f_{2}$ might intersect $E$. If this happens, the functions $f_{1}$ and $f_{2}$ should be approximated by functions from $\mathcal{D}_{E}(G)$, see [32, Remark 4] for the details.

Remark 3.1. (i) In Theorem 3.2, the assumption of 1-UCP with pointwise unconditionally convergence of the Fourier series of $w_{f}$ can be replaced by the simpler, but stronger, assumption that $\cup_{j \in J}\left\{T_{\gamma} g_{j, p}\right\}_{\gamma \in \Gamma_{j}, p \in P_{j}}$ satisfies that $\infty$-UCP.

(ii) While the existence of a $D^{\prime}$-sequence in $\widehat{G}$ is sufficient for the differentiation process on Lebesgue's integrals [29, Theorem 44.18], it may not be necessary. In fact, it is an open problem whether Lebesgue's differentiation theorem holds on all second countable locally compact abelian groups [8, Section 7.1].

The 1-UCP assumption in Theorem 3.2 cannot be dropped as demonstrated by Example 3.2 below. The construction of the generalized shift-invariant system follows [9, Example 3.2].

Example 3.2. Let $G=\mathbb{Z}$. Let $N \in \mathbb{N}$ be such that $N \geq 2$. Define the lattices $\Gamma_{j}=N^{j} \mathbb{Z}$ for $j \in \mathbb{N}$. Let $\tau_{1}=0$ and define $\tau_{j}, j \geq 2$, inductively as the smallest $t \in \mathbb{Z}$ in absolute value satisfying

$$
\left(\bigcup_{i=1}^{j-1}\left(\tau_{i}+N^{i} \mathbb{Z}\right)\right) \cap\left(t+N^{j} \mathbb{Z}\right)=\emptyset
$$


In case $t$ and $-t$ both are minimizers, pick $\tau_{j}$ to be positive. Then

$$
\mathbb{Z}=\biguplus_{j \in \mathbb{N}}\left(\tau_{j}+N^{j} \mathbb{Z}\right)
$$

with the union being disjoint, see also [24, Lemma 4.4]. Define the generators $g_{j}=\mathbb{1}_{\tau_{j}} \in \ell^{2}(\mathbb{Z})$ for $j \in \mathbb{N}$. By construction, the generalized shift-invariant system $\cup_{j \in \mathbb{N}}\left\{T_{\gamma} g_{j}\right\}_{\gamma \in \Gamma_{j}}$ is the canonical basis $\left\{\mathbb{1}_{k}\right\}_{k \in \mathbb{Z}}$ for $\ell^{2}(\mathbb{Z})$ and thus it forms, in particular, a frame for $\ell^{2}(\mathbb{Z})$ with frame bounds $A=B=1$. The system $\cup_{j \in \mathbb{N}}\left\{T_{\gamma} g_{j}\right\}_{\gamma \in \Gamma_{j}}$ satisfies the 1-UCP only for the case $N=2$ as shown in [24, Example 1]. We now show that the bound (3.2) fails for $N \geq 3$ in spite of the Bessel property.

Observe that $\hat{g}_{j} \in L^{2}(\mathbb{T})$ is given by $\hat{g}_{j}(\omega)=e^{2 \pi i \tau_{j} \omega}$ for $\omega \in[0,1)$. Hence, for any $\alpha \in \bigcup_{j=1}^{\infty} N^{-j} \mathbb{Z}$

$$
t_{\alpha}(\omega)=\sum_{j \in \mathbb{N}: \alpha \in N^{-j} \mathbb{Z}} \frac{1}{\mathrm{~d}\left(\Gamma_{j}\right)} \hat{g}_{j}(\omega) \overline{\hat{g}_{j}(\omega+\alpha)}=\sum_{j \in \mathbb{N}: \alpha \in N^{-j} \mathbb{Z}} N^{-j} \mathrm{e}^{-2 \pi i \alpha \tau_{j}}
$$

for all $\omega \in[0,1)$. Since $t_{\alpha}$ is independent of the variable $\omega \in[0,1)$, we fix an arbitrary $\omega \in[0,1)$ in the following calculations.

We rewrite $\bigcup_{j=1}^{\infty} N^{-j} \mathbb{Z}$ as the following disjoint union:

$$
\bigcup_{j=1}^{\infty} N^{-j} \mathbb{Z}=\mathbb{Z} \bigcup\left(\bigcup_{m=1}^{\infty} N^{-m}(\mathbb{Z} \backslash N \mathbb{Z})\right) .
$$

For $\alpha \in \mathbb{Z}$, a direct calculation gives

$$
t_{\alpha}(\omega)=\sum_{j=1}^{\infty} N^{-j}=\frac{1}{N-1} .
$$

On the other hand, writing $\alpha \notin \mathbb{Z}$ as $\alpha=k N^{-m}$ for $k \in \mathbb{Z} \backslash N \mathbb{Z}$ and $m \in \mathbb{N}$ yields

$$
t_{\alpha}(\omega)=\sum_{j=m}^{\infty} N^{-j} \mathrm{e}^{-2 \pi i k N^{-m} \tau_{j}} .
$$

Hence

$$
\left|t_{\alpha}(\omega)\right|=N^{-m}\left|\sum_{\ell=0}^{\infty} N^{-\ell} \mathrm{e}^{-2 \pi i k N^{-m}} \tau_{\ell+m}\right| .
$$

Next, we claim that it then follows that

$$
\left|t_{\alpha}(\omega)\right| \geq N^{-m}\left(1-\sum_{\ell=1}^{\infty} N^{-\ell}\right)=N^{-m} \frac{N-2}{N-1} .
$$

To see this claim, let $z_{\ell} \in \mathbb{T}, \ell \in \mathbb{N}_{0}$. By the triangle inequality, we have

$$
\left|\sum_{\ell=0}^{\infty} N^{-\ell} z_{\ell}\right| \geq\left|z_{0}\right|-\left|z_{0}-\sum_{\ell=0}^{\infty} N^{-\ell} z_{\ell}\right|=1-\left|\sum_{\ell=1}^{\infty} N^{-\ell} z_{\ell}\right| \geq 1-\sum_{\ell=1}^{\infty} N^{-\ell}=\frac{N-2}{N-1},
$$

which proves the claim. 
Let $N \geq 3$. Since for each $m \in \mathbb{N}$ there are infinitely many $k \in \mathbb{Z} \backslash N \mathbb{Z}$ with $\left|t_{k N^{-m}}(\omega)\right|>$ $N^{-m} / 2$, we see that (3.2) is violated as $B_{2}=\infty$. In fact, for any $p \in[1, \infty)$, we have

$$
\sum_{\alpha \in \bigcup_{j \in J} \Gamma_{j}^{\perp}}\left|t_{\alpha}(\omega)\right|^{p}=\infty
$$

for all $\omega \in[0,1)$. Thus, no $\ell^{p}$-norm of $\left\{t_{\alpha}(\omega)\right\}_{\alpha \in \bigcup_{j \in J} \Gamma_{j}^{\perp}}$, with $\omega \in[0,1)$, can be finite.

The discreteness of the group $G=\mathbb{Z}$ in Example 3.2 is not crucial. In fact, the construction is easily transferred to $L^{2}(\mathbb{R})$, e.g., by starting from the Gabor-like orthonormal basis $\left\{T_{k} \mathrm{e}^{2 \pi i m \cdot} \mathbb{1}_{[0,1)}\right\}_{k, m \in \mathbb{Z}} \cdot$

\subsection{Sufficient conditions}

The following result, Theorem [3.3, provides a sufficient condition and estimates of the frame bounds for generalized translation-invariant frames. The proof of Theorem 3.3 is based on the simple estimate that a (generalized) Fourier series of an almost periodic function is bounded from above by the sum of the modulus of its coefficients and is bounded from below by the absolute value of its constant term minus the sum of the other terms in modulus.

Theorem 3.3. Let $\cup_{j \in J}\left\{T_{\gamma} g_{j, p}\right\}_{\gamma \in \Gamma_{j}, p \in P_{j}}$ be a generalized translation-invariant system satisfying the 1-UCP.

(i) Suppose $\cup_{j \in J}\left\{T_{\gamma} g_{j, p}\right\}_{\gamma \in \Gamma_{j}, p \in P_{j}}$ satisfies

$$
B_{1}:=\operatorname{esssup}_{\omega \in \widehat{G}} \sum_{\alpha \in \bigcup_{j \in J} \Gamma_{j}^{\perp}}\left|t_{\alpha}(\omega)\right|<\infty
$$

then $\cup_{j \in J}\left\{T_{\gamma} g_{j, p}\right\}_{\gamma \in \Gamma_{j}, p \in P_{j}}$ forms a Bessel system in $L^{2}(G)$ with Bessel bound $B_{1}$.

(ii) Suppose $\cup_{j \in J}\left\{T_{\gamma} g_{j, p}\right\}_{\gamma \in \Gamma_{j}, p \in P_{j}}$ satisfies (3.10) and

$$
A_{1}:=\underset{\omega \in \widehat{G}}{\operatorname{essinf}}\left(t_{0}(\omega)-\sum_{\alpha \in \bigcup_{j \in J} \Gamma_{j}^{\perp} \backslash\{0\}}\left|t_{\alpha}(\omega)\right|\right)>0,
$$

then $\cup_{j \in J}\left\{T_{\gamma} g_{j, p}\right\}_{\gamma \in \Gamma_{j}, p \in P_{j}}$ forms a frame for $L^{2}(G)$ with lower bound $A_{1}$ and upper bound $B_{1}$.

Proof. Suppose the system $\cup_{j \in J}\left\{T_{\gamma} g_{j, p}\right\}_{\gamma \in \Gamma_{j}, p \in P_{j}}$ satisfies condition (3.10) and the 1-UCP with respect to $E \in \mathcal{E}$. By definition of $w_{f}$ and the fact that $\mathcal{D}_{E}(G)$ is dense in $L^{2}(G)$, it suffices in (ii) to show that $A_{1}\|f\|^{2} \leq w_{f}(0) \leq B_{1}\|f\|^{2}$ for all $f \in \mathcal{D}_{E}(G)$, while in (i) it suffices to prove the upper bound.

As a consequence of Proposition 2.3, the auxiliary function $w_{f}: G \rightarrow \mathbb{C}$ possesses the generalized Fourier series $\sum_{\alpha \in \bigcup_{j \in J} \Gamma_{j}^{\perp}} c_{\alpha} \alpha$, where $c_{\alpha}:=\int_{\widehat{G}} \hat{f}(\omega) \overline{\hat{f}(\omega+\alpha)} \overline{t_{\alpha}(\omega)} d \mu_{\widehat{G}}(\omega)$. It will first be shown that $w_{f}$ coincides point-wise with its generalized Fourier series. In order to do this, we show that the generalized Fourier series is uniformly convergent. An application of Beppo Levi's theorem and Young's inequality for products gives

$$
\sum_{\alpha \in \bigcup_{j \in J} \Gamma_{j}^{\perp} \backslash\{0\}}\left|c_{\alpha}\right| \leq \int_{\widehat{G}} \sum_{\alpha \in \bigcup_{j \in J} \Gamma_{j}^{\perp} \backslash\{0\}}\left|\hat{f}(\omega) \hat{f}(\omega+\alpha) t_{\alpha}(\omega)\right| d \mu_{\widehat{G}}(\omega)
$$




$$
\begin{aligned}
& \leq \frac{1}{2} \int_{\widehat{G}}|\hat{f}(\omega)|^{2} \sum_{\alpha \in \bigcup_{j \in J} \Gamma_{j}^{\perp} \backslash\{0\}}\left|t_{\alpha}(\omega)\right| d \mu_{\widehat{G}}(\omega) \\
& \quad+\frac{1}{2} \int_{\widehat{G}} \sum_{\alpha \in \bigcup_{j \in J} \Gamma_{j}^{\perp} \backslash\{0\}}|\hat{f}(\omega+\alpha)|^{2}\left|t_{\alpha}(\omega)\right| d \mu_{\widehat{G}}(\omega) \\
& =\int_{\widehat{G}}|\hat{f}(\omega)|^{2} \sum_{\alpha \in \bigcup_{j \in J} \Gamma_{j}^{\perp} \backslash\{0\}}\left|t_{\alpha}(\omega)\right| d \mu_{\widehat{G}}(\omega),
\end{aligned}
$$

where equality follows by the change of variable $\omega \mapsto \omega-\alpha$ and $\overline{t_{\alpha}(\omega-\alpha)}=t_{-\alpha}(\omega)$. Since $\sum_{\alpha \in \bigcup_{j \in J} \Gamma_{j}^{\perp}}\left|c_{\alpha}\right|<\infty$, an application of the Weierstrass M-test yields that the generalized Fourier series of $w_{f}$ converges uniformly to an almost periodic function. By uniqueness of Fourier coefficients, it follows that $w_{f}(x)=\sum_{\alpha \in \bigcup_{j \in J} \Gamma_{j}^{\perp}} c_{\alpha} \alpha(x)$ pointwise for all $x \in G$.

Now, setting $x=0$ in the Fourier series representation of $w_{f}$ and using (3.12) give

$$
w_{f}(0)=\sum_{\alpha \in \cup_{j \in J} \Gamma_{j}^{\perp}} c_{\alpha} \leq \int_{\widehat{G}}|\hat{f}(\omega)|^{2} \sum_{\alpha \in \cup_{j \in J} \Gamma_{j}^{\perp}}\left|t_{\alpha}(\omega)\right| d \mu_{\widehat{G}}(\omega) \leq B_{1}\|f\|_{2}^{2}
$$

for all $f \in \mathcal{D}_{E}(G)$. This shows (i). Assume now also that the assumption in (ii) is satisfied. Then, by the triangle inequality and (3.12), we have for all $f \in \mathcal{D}_{E}(G)$

$$
\begin{aligned}
w_{f}(0) & =\sum_{\alpha \in \cup_{j \in J} \Gamma_{j}^{\perp}} c_{\alpha} \geq c_{0}-\left|\sum_{\alpha \in \bigcup_{j \in J} \Gamma_{j}^{\perp} \backslash\{0\}} c_{\alpha}\right| \\
& \geq \int_{\widehat{G}}|\hat{f}(\omega)|^{2}\left(t_{0}(\omega)-\sum_{\alpha \in \bigcup_{j \in J} \Gamma_{j}^{\perp} \backslash\{0\}}\left|t_{\alpha}(\omega)\right|\right) d \mu_{\widehat{G}}(\omega) \geq A_{1}\|f\|_{2}^{2}
\end{aligned}
$$

as desired.

The frame bound estimates of Theorem 3.3 are optimal for tight frames. That is, for a generalized translation-invariant system $\cup_{j \in J}\left\{T_{\gamma} g_{j, p}\right\}_{\gamma \in \Gamma_{j}, p \in P_{j}}$ that satisfies the 1-UCP and forms a tight frame, the estimates in Theorem 3.3 recover precisely the frame bound of the given frame. This simple observation is stated as the next result.

Proposition 3.4. Let $\cup_{j \in J}\left\{T_{\gamma} g_{j, p}\right\}_{\gamma \in \Gamma_{j}, p \in P_{j}}$ be a generalized translation-invariant system satisfying the 1-UCP. Suppose $\cup_{j \in J}\left\{T_{\gamma} g_{j, p}\right\}_{\gamma \in \Gamma_{j}, p \in P_{j}}$ forms a tight frame for $L^{2}(G)$ with frame bound $A>0$. Then $A=A_{1}=B_{1}$.

Proof. Suppose the system $\cup_{j \in J}\left\{T_{\gamma} g_{j, p}\right\}_{\gamma \in \Gamma_{j}, p \in P_{j}}$ is a tight frame for $L^{2}(G)$ with bound $A>0$. By [32, Theorem 3.4] and [24, Theorem 3.11], it holds, for any $\alpha \in \bigcup_{j \in J} \Gamma_{j}^{\perp}$, that

$$
t_{\alpha}(\omega)=A \delta_{\alpha, 0}
$$

for $\mu_{\widehat{G}^{-}}$-a.e. $\omega \in \widehat{G}$. Hence $\sum_{\alpha \in \bigcup_{j \in J} \Gamma_{j}^{\perp} \backslash\{0\}}\left|t_{\alpha}(\omega)\right|=0$ almost everywhere on $\widehat{G}$ and the conclusion follows. 


\subsection{Comparison of frame bound estimates}

In this section we compare the frame bound estimates provided by Theorem 3.3 with known estimates. For this, we state the following result [32, Proposition 3.7].

Proposition 3.5. Let $\cup_{j \in J}\left\{T_{\gamma} g_{j, p}\right\}_{\gamma \in \Gamma_{j}, p \in P_{j}}$ be a generalized translation-invariant system.

(i) Suppose the system $\cup_{j \in J}\left\{T_{\gamma} g_{j, p}\right\}_{\gamma \in \Gamma_{j}, p \in P_{j}}$ satisfies

$$
B^{\prime}:=\operatorname{esssup}_{\omega \in \widehat{G}} \sum_{j \in J} \frac{1}{\mathrm{~d}\left(\Gamma_{j}\right)} \int_{P_{j}} \sum_{\alpha \in \Gamma_{j}^{\perp}}\left|\hat{g}_{j, p}(\omega) \hat{g}_{j, p}(\omega+\alpha)\right| d \mu_{P_{j}}(p)<\infty,
$$

then $\cup_{j \in J}\left\{T_{\gamma} g_{j, p}\right\}_{\gamma \in \Gamma_{j}, p \in P_{j}}$ forms a Bessel family in $L^{2}(G)$ with Bessel bound $B^{\prime}$.

(ii) Suppose the system $\cup_{j \in J}\left\{T_{\gamma} g_{j, p}\right\}_{\gamma \in \Gamma_{j}, p \in P_{j}}$ satisfies and

$$
\begin{aligned}
A^{\prime}:=\underset{\omega \in \widehat{G}}{\operatorname{essinf}}( & \sum_{j \in J} \frac{1}{\mathrm{~d}\left(\Gamma_{j}\right)} \int_{P_{j}}\left|\hat{g}_{j, p}(\omega)\right|^{2} d \mu_{P_{j}}(p) \\
& \left.-\sum_{j \in J} \frac{1}{\mathrm{~d}\left(\Gamma_{j}\right)} \int_{P_{j}} \sum_{\alpha \in \Gamma_{j}^{\perp} \backslash\{0\}}\left|\hat{g}_{j, p}(\omega) \hat{g}_{j, p}(\omega+\alpha)\right| d \mu_{P_{j}}(p)\right)>0,
\end{aligned}
$$

then $\cup_{j \in J}\left\{T_{\gamma} g_{j, p}\right\}_{\gamma \in \Gamma_{j}, p \in P_{j}}$ forms a frame for $L^{2}(G)$ with lower bound $A^{\prime}$ and upper bound $B^{\prime}$.

In [32], the term absolute CC-condition was used for condition (3.13). The important difference between the CC-condition (3.10) and the absolute CC-condition (3.13) is the placement of the absolute sign in the summand. In the CC-condition, it is possible to have phase cancellations within each auto-correlation function while the absolute CC-condition prohibits such cancellations. It is a simple observation that a generalized translation-invariant system satisfying the absolute CC-condition also satisfies the CC-condition.

Lemma 3.6. Suppose $\cup_{j \in J}\left\{T_{\gamma} g_{j, p}\right\}_{\gamma \in \Gamma_{j}, p \in P_{j}}$ satisfies the absolute $C C$-condition (3.13). Then $\cup_{j \in J}\left\{T_{\gamma} g_{j, p}\right\}_{\gamma \in \Gamma_{j}, p \in P_{j}}$ also satisfies the $C C$-condition (3.10).

Proof. Suppose the system $\cup_{j \in J}\left\{T_{\gamma} g_{j, p}\right\}_{\gamma \in \Gamma_{j}, p \in P_{j}}$ satisfies the absolute CC-condition (3.13). Then an application of Beppo Levi's theorem gives

$$
\begin{aligned}
& \sum_{j \in J} \sum_{\alpha \in \Gamma_{j}^{\perp}}\left|\frac{1}{\mathrm{~d}\left(\Gamma_{j}\right)} \int_{P_{j}} \hat{g}_{j, p}(\omega) \overline{\hat{g}_{j, p}(\omega+\alpha)} d \mu_{P_{j}}(p)\right| \\
& \leq \sum_{j \in J} \frac{1}{\mathrm{~d}\left(\Gamma_{j}\right)} \int_{P_{j}} \sum_{\alpha \in \Gamma_{j}^{\perp}}\left|\hat{g}_{j, p}(\omega) \hat{g}_{j, p}(\omega+\alpha)\right| d \mu_{P_{j}}(p)<\infty
\end{aligned}
$$

for $\mu_{\widehat{G}^{-}}$a.e. $\omega \in \widehat{G}$. Using the absolute convergence of the series, a re-ordering of the summation does not affect the convergence. Thus

$$
\operatorname{essup}_{\omega \in \widehat{G}} \sum_{\alpha \in \bigcup_{j \in J} \Gamma_{j}^{\perp}}\left|\sum_{j \in J: \alpha \in \Gamma_{j}^{\perp}} \frac{1}{\mathrm{~d}\left(\Gamma_{j}\right)} \int_{P_{j}} \hat{g}_{j, p}(\omega) \overline{\hat{g}_{j, p}(\omega+\alpha)} d \mu_{P_{j}}(p)\right|<\infty,
$$

as required. 
Jakobsen and the first named author [32] show that a generalized translation-invariant system satisfying the absolute CC-condition automatically satisfies the $\alpha$-LIC. Thus the 1 UCP is implicitly assumed in the estimate (3.13).

The generalized translation-invariant system in Example 3.2 with $N=2$ satisfies the frame bound estimates based on the CC-condition, but dramatically fails the estimates based on the absolute CC-condition as demonstrated in the next example.

Example 3.3. Let $G=\mathbb{Z}$. Consider the system $\cup_{j \in \mathbb{N}}\left\{T_{\gamma} g_{j}\right\}_{\gamma \in \Gamma_{j}}$ in $\ell^{2}(\mathbb{Z})$ with $\Gamma_{j}=2^{j} \mathbb{Z}$ and $g_{j}=\mathbb{1}_{\tau_{j}}$, where $\left(\tau_{j}\right)_{j \in \mathbb{N}} \subset \mathbb{Z}$ is chosen as in (3.9). This system forms a frame for $\ell^{2}(\mathbb{Z})$ with frame bounds $A=B=1$. For $\omega \in[0,1)$, a direct calculation gives

$$
\sum_{j \in \mathbb{N}} \frac{1}{\mathrm{~d}\left(\Gamma_{j}\right)} \sum_{\alpha \in \Gamma_{j}^{\perp}}\left|\hat{g}_{j}(\omega) \hat{g}_{j}(\omega+\alpha)\right|=\sum_{j \in \mathbb{N}} \frac{1}{2^{j}} \sum_{\alpha \in \Gamma_{j}^{\perp}}\left|e^{2 \pi i \tau_{j} \alpha}\right|=\sum_{j \in \mathbb{N}} \frac{1}{2^{j}} \#\left(\Gamma_{j}^{\perp}\right)=\sum_{j \in \mathbb{N}} 1=\infty .
$$

Thus $\cup_{j \in \mathbb{N}}\left\{T_{\gamma} g_{j}\right\}_{\gamma \in \Gamma_{j}}$ fails the estimate (3.13). On the other hand, it follows by Proposition 3.4 that $\cup_{j \in \mathbb{N}}\left\{T_{\gamma} g_{j}\right\}_{\gamma \in \Gamma_{j}}$ satisfies the estimates (3.10) and (3.11) with bounds $A_{1}=B_{1}=1$.

The discrepancy between the frame bound estimates in Proposition 3.5 and the estimates in Theorem 3.3 might occur even for well-known orthonormal bases. Indeed, the Meyer wavelet is an example of an orthonormal basis in $L^{2}(\mathbb{R})$ for which the estimates based on the absolute CC-condition give the poor estimates $A^{\prime}=-1$ and $B^{\prime}=3$, see [19, p. 984]. However, the frame bound estimates in Theorem 3.3 give the correct frame bounds, namely $A_{1}=B_{1}=1$. For a direct verification of the characterizing equations $t_{\alpha}=\delta_{\alpha, 0}$ for the Meyer wavelet, the interested reader to Daubechies' book [20, Section 4.2.1].

Finally, we remark that Casazza, Christensen and Janssen [13] give an example of a Gabor system forming a Bessel system in $L^{2}(\mathbb{R})$, but where $\sum_{\alpha}\left|t_{\alpha}(\omega)\right|=\infty$ for a.e. $\omega \in \mathbb{R}$. This demonstrates that for Bessel systems both the CC-condition and the absolute CC-condition can fail even though the LIC and thus $\alpha$-LIC and the 1-UCP hold.

\section{Applications and examples}

In this section the sufficient conditions given in Theorem 3.3 will be considered for special types of generalized translation-invariant systems. In the examples presented in this section, the focus will be on explicit formulas for the auto-correlation functions $t_{\alpha}: \widehat{G} \rightarrow \mathbb{C}$ and the associated remainder function

$$
R: \widehat{G} \rightarrow[0, \infty], \quad R(\omega)=\sum_{\alpha \in \bigcup_{j \in J} \Gamma_{j}^{\perp} \backslash\{0\}}\left|t_{\alpha}(\omega)\right|,
$$

which are the main ingredients in the estimates in Theorem 3.3. Here, it should be understood that the (formal) expression for $t_{\alpha}$ might only be well-defined once we impose the CC-condition. Stating the necessary condition in Theorem 3.2 in each special case from these formulas is straightforward and is left to the reader.

\subsection{Gabor systems}

Given a countable index set $J$, let $\left\{g_{j}\right\}_{j \in J} \subset L^{2}(G)$. Let $\Gamma \subseteq G$ be a closed, co-compact subgroup and let $\Lambda \subseteq \widehat{G}$ be such that equipping it with a $\sigma$-algebra $\Sigma_{\Lambda}$ and a measure $\mu_{\Lambda}$ gives a measure space $\left(\Lambda, \Sigma_{\Lambda}, \mu_{\Lambda}\right)$ satisfying the standard hypotheses. The (semi) co-compact Gabor system associated with the pair $(\Gamma, \Lambda)$ is the collection of functions

$$
\left\{M_{\lambda} T_{\gamma} g_{j}\right\}_{\lambda \in \Lambda, \gamma \in \Gamma, j \in J}=\left\{\lambda(\cdot) g_{j}(\cdot-\gamma)\right\}_{\lambda \in \Lambda, \gamma \in \Gamma, j \in J}
$$


where $M_{\lambda} f(x)=\lambda(x) f(x)$ denotes the modulation operator on $L^{2}(G)$. The Gabor system $\left\{M_{\lambda} T_{\gamma} g_{j}\right\}_{\lambda \in \Lambda, \gamma \in \Gamma, j \in J}$ cannot be expressed as a generalized translation-invariant system. However, since $\left|\left\langle f, M_{\lambda} T_{\gamma} g_{j}\right\rangle\right|=\left|\left\langle f, T_{\gamma} M_{\lambda} g_{j}\right\rangle\right|$ for all $f \in L^{2}(G)$, the Gabor system $\left\{M_{\lambda} T_{\gamma} g_{j}\right\}_{\lambda \in \Lambda, \gamma \in \Gamma, j \in J}$ forms a Bessel system or a frame if, and only if, the corresponding (generalized) translation-invariant system $\left\{T_{\gamma} M_{\lambda} g_{j}\right\}_{\lambda \in \Lambda, \gamma \in \Gamma, j \in J}$ with $g_{j, \lambda}=M_{\lambda} g_{j}$ forms a Bessel system or a frame. The auto-correlation functions $t_{\alpha}$ associated with $\left\{T_{\gamma} M_{\lambda} g_{j}\right\}_{\lambda \in \Lambda, \gamma \in \Gamma, j \in J}$ can (formally) be written as

$$
t_{\alpha}(\omega)=\sum_{j \in J} \int_{\Lambda} \hat{g}_{j}(\omega-\lambda) \overline{\hat{g}_{j}(\omega-\lambda-\alpha)} d \mu_{\Lambda}(\lambda)
$$

for $\alpha \in \Gamma^{\perp}$. Any translation-invariant system satisfying the CC-condition also satisfies the $\alpha$-LIC. Thus an application of Theorem 3.3 gives the frame bound estimates (3.10) and (3.11), where the 0th auto-correlation function $t_{0}$ is given by

$$
t_{0}(\omega)=\sum_{j \in J} \int_{\Lambda}\left|\hat{g}_{j}(\omega-\lambda)\right|^{2} d \mu_{\Lambda}(\lambda)
$$

and the remainder function $R: \widehat{G} \rightarrow[0, \infty]$ by

$$
R(\omega)=\sum_{\alpha \in \Gamma^{\perp} \backslash\{0\}}\left|\sum_{j \in J} \int_{\Lambda} \hat{g}_{j}(\omega-\lambda) \overline{\hat{g}_{j}(\omega-\lambda-\alpha)} d \mu_{\Lambda}(\lambda)\right| .
$$

The frame bound estimates associated with (4.1) and (4.2) allow for phase cancellations over the modulation parameter $\lambda \in \Lambda$. Moreover, if $\Lambda$ is a closed subgroup, we only need to take the essential supremum and infimum in (3.10) and (3.11), respectively, over a fundamental domain of $\Lambda$ in $\widehat{G}$. For singly generated Gabor frames in $L^{2}\left(\mathbb{R}^{d}\right)$ associated with a pair of full-rank lattices $(\Lambda, \Gamma)$, the frame bound estimates (3.10) and (3.11) using (4.1) and (4.2) recover precisely the frame bound estimates by Ron and Shen [44, 48].

The sufficient conditions for Gabor frames are often formulated in the time domain. To do this, we switch the role of $\Gamma$ and $\Lambda$ and consider the Gabor system $\left\{M_{\lambda} T_{\gamma} g_{j}\right\}_{\lambda \in \Lambda, \gamma \in \Gamma, j \in J}=$ $\left\{T_{\lambda} \mathcal{F}^{-1} T_{\gamma} g_{j}\right\}_{\lambda \in \Lambda, \gamma \in \Gamma, j \in J}$, where $\mathcal{F}^{-1}$ denotes the inverse Fourier transform. In this way, one obtains auto-correlation functions $s_{\alpha}: G \rightarrow \mathbb{C}, \alpha \in \Lambda^{\perp}$, given by

$$
s_{\alpha}(x):=\sum_{j \in J} \int_{\Gamma} \overline{g_{j}(x-\gamma-\alpha)} g_{j}(x-\gamma) d \mu_{\Gamma}(\gamma),
$$

provided the series converges. Hence, if

$$
B_{1}:=\underset{x \in G}{\operatorname{esssup}} \sum_{\alpha \in \Lambda^{\perp}}\left|s_{\alpha}(x)\right|<\infty
$$

and

$$
A_{1}:=\underset{x \in G}{\operatorname{essinf}}\left(s_{0}(x)-\sum_{\alpha \in \Gamma^{\perp} \backslash\{0\}}\left|s_{\alpha}(x)\right|\right)>0,
$$

then $\left\{M_{\lambda} T_{\gamma} g_{j}\right\}_{\lambda \in \Lambda, \gamma \in \Gamma, j \in J}$ is a frame for $L^{2}\left(\mathbb{R}^{d}\right)$ with bounds $A_{1}$ and $B_{1}$. For singly generated Gabor frames in $L^{2}\left(\mathbb{R}^{d}\right)$ associated with a pair of full-rank lattices $(\Lambda, \Gamma)$, these estimates recover precisely [25, Proposition 6.5.5]. 


\subsection{Wavelet systems}

Let $\operatorname{Aut}(G)$ denote the collection of all bi-continuous group homomorphisms on $G$. For an automorphism $a \in \operatorname{Aut}(G)$, let $|a|$ denote its modulus, i.e., the unique positive constant such that

$$
\int_{G} f(a(x)) d \mu_{G}(x)=|a| \int_{G} f(x) d \mu_{G}(x)
$$

for all $f \in L^{1}(G)$. Denote by $D_{a} f(x):=|a|^{1 / 2} f(a(x))$ the unitary dilation operator on $L^{2}(G)$.

Let $J$ and $L$ be countable index sets, let $\left\{\psi_{\ell}\right\}_{\ell \in L} \subset L^{2}(G)$, let $\mathcal{A}:=\left\{a_{j}\right\}_{j \in J} \subset \operatorname{Aut}(G)$ and let $\Gamma \subseteq G$ be a closed, co-compact subgroup. The wavelet system in $L^{2}(G)$, associated with the pair $(\mathcal{A}, \Gamma)$, is the collection of functions

$$
\left\{D_{a} T_{\gamma} \psi_{\ell}\right\}_{a \in \mathcal{A}, \gamma \in \Gamma, \ell \in L}=\left\{\left|a_{j}\right|^{1 / 2} \psi_{\ell}\left(a_{j}(\cdot)-\gamma\right)\right\}_{j \in J, \gamma \in \Gamma, \ell \in L} .
$$

By considering the commutation relation $D_{a} T_{\gamma}=T_{a^{-1}(\gamma)} D_{a}$ for $a \in \mathcal{A}$ and $\gamma \in \Gamma$, the wavelet system (4.3) can be written as the generalized translation-invariant system $\cup_{j \in J}\left\{T_{\gamma} g_{j, p}\right\}_{\gamma \in \Gamma_{j}}$ with $\Gamma_{j}=a_{j}^{-1}(\Gamma)$ and $g_{j, p}=D_{a_{j}} \psi_{\ell}$ for $j \in J$ and $p=\ell \in P$ with $P=L$ equipped with the counting measure.

The adjoint of an automorphism $a \in \operatorname{Aut}(G)$ is the automorphism $\hat{a}: \widehat{G} \rightarrow \widehat{G}$ defined by $\hat{a}(\omega)=\omega \circ a$ for $\omega \in \widehat{G}$. Using this notion, the annihilators $\Gamma_{j}^{\perp}$ of $\Gamma_{j}$ for $j \in J$ can be written as $\Gamma_{j}^{\perp}=\left(a_{j}^{-1}(\Gamma)\right)^{\perp}=\hat{a}_{j}\left(\Gamma^{\perp}\right)$, cf. [8, Proposition 6.5]. For $\alpha \in \bigcup_{j \in J} \hat{a}_{j}\left(\Gamma^{\perp}\right)$, the auto-correlation function $t_{\alpha}: \widehat{G} \rightarrow \mathbb{C}$ can be formally written as

$$
\begin{aligned}
t_{\alpha}(\omega) & =\sum_{\ell \in L} \sum_{j \in \kappa(\alpha)} \frac{1}{\mathrm{~d}\left(\Gamma_{j}\right)}\left(\widehat{D_{a_{j}} \psi_{\ell}}\right)(\omega) \overline{\left(\widehat{D_{a_{j}} \psi_{\ell}}\right)(\omega+\alpha)} \\
& =\sum_{\ell \in L} \sum_{j \in \kappa(\alpha)} \frac{\left|a_{j}\right|}{\mathrm{d}\left(\Gamma_{j}\right)} \hat{\psi}_{\ell}\left(\hat{a}_{j}^{-1}(\omega)\right) \overline{\hat{\psi}_{\ell}\left(\hat{a}_{j}^{-1}(\omega+\alpha)\right)}
\end{aligned}
$$

where $\kappa(\alpha):=\left\{j \in J \mid \alpha \in \hat{a}_{j}\left(\Gamma^{\perp}\right)\right\}$. Observe that $\kappa(0)=J$. Therefore, for wavelet systems satisfying the 1-UCP, an application of Theorem 3.3 yields the frame bound estimates as in (3.10) and (3.11), where

$$
t_{0}(\omega)=\sum_{\ell \in L} \sum_{j \in \kappa(\alpha)} \frac{\left|a_{j}\right|}{\mathrm{d}\left(\Gamma_{j}\right)}\left|\hat{\psi}_{\ell}\left(\hat{a}_{j}^{-1}(\omega)\right)\right|^{2}
$$

is the Calderón sum, and the remainder function $R: \widehat{G} \rightarrow[0, \infty]$ takes the form

$$
R(\omega)=\sum_{\alpha \in \bigcup_{j \in J}}\left|\sum_{\hat{a}_{j}\left(\Gamma^{\perp}\right) \backslash\{0\}} \sum_{\ell \in L} \frac{\left|a_{j}\right|}{\mathrm{d}\left(\Gamma_{j}\right)} \hat{\psi}_{\ell}\left(\hat{a}_{j}^{-1}(\omega)\right) \overline{\hat{\psi}_{\ell}\left(\hat{a}_{j}^{-1}(\omega+\alpha)\right)}\right| .
$$

Thus for all generators and for all scales in $\kappa(\alpha)$, we have the possibility of cancellations in the estimates for each $\alpha \in \bigcup_{j \in J} \hat{a}_{j}\left(\Gamma^{\perp}\right) \backslash\{0\}$. This possibility of cancellations is in contrast to known sufficient conditions and frame bound estimates for wavelet systems based on the absolute CC-condition. These latter sufficient conditions use the remainder function $\tilde{R}: \widehat{G} \rightarrow$ $[0, \infty]$ given by

$$
\tilde{R}(\omega)=\sum_{j \in J} \frac{\left|a_{j}\right|}{\mathrm{d}\left(\Gamma_{j}\right)} \sum_{\alpha \in \hat{a}_{j}\left(\Gamma^{\perp}\right) \backslash\{0\}} \sum_{\ell \in L}\left|\hat{\psi}_{\ell}\left(\hat{a}_{j}^{-1} \omega\right) \overline{\hat{\psi}_{\ell}\left(\hat{a}_{j}^{-1}(\omega+\alpha)\right)}\right|,
$$

in which only the modulus of the generating functions are considered. To wrap up the discussion, we state the following result. 
Theorem 4.1. Given countable index sets $J, L$, let $\left\{\psi_{\ell}\right\}_{\ell \in L} \subset L^{2}(G)$, let $\left\{a_{j}\right\}_{j \in J} \subset \operatorname{Aut}(G)$ and let $\Gamma \subseteq G$ be a closed, co-compact subgroup. Suppose the system $\left\{D_{a_{j}} T_{\gamma} \psi_{\ell}\right\}_{j \in J, \gamma \in \Gamma, \ell \in L}$ satisfies the 1-UCP and satisfies

$$
b_{1}:=\operatorname{esssup}_{\omega \in \widehat{G}}\left(t_{0}(\omega)+R(\omega)\right)<\infty
$$

and

$$
a_{1}:=\underset{\omega \in \widehat{G}}{\operatorname{essinf}}\left(t_{0}(\omega)-R(\omega)\right)>0,
$$

where $t_{0}$ and $R$ are given in (4.4) and (4.5), respectively. Then $\left\{D_{a_{j}} T_{\gamma} \psi_{\ell}\right\}_{j \in J, \gamma \in \Gamma, \ell \in L}$ forms a frame for $L^{2}(G)$ with bounds $a_{1}$ and $b_{1}$.

The wavelet system in (4.3) is defined with respect to an arbitrary family of automorphisms $\mathcal{A} \subseteq \operatorname{Aut}(G)$. For such general systems, the LIC, and hence $\alpha$-LIC and 1-UCP, are not necessarily satisfied whenever the system satisfies the CC-condition. However, under additional assumptions on the family $\mathcal{A} \subseteq \operatorname{Aut}(G)$, simple sufficient conditions and characterizations for the LIC are known. For example, for a family $\left\{a_{j}\right\}_{j \in J} \subset \operatorname{Aut}(G)$ for which the adjoints $\left\{\hat{a}_{j}\right\}_{j \in J}$ are expanding in the sense of [1, Definition 18], the LIC is automatically satisfied for any system satisfying the CC-condition. In particular, for a cyclic group $\mathcal{A}=\langle a\rangle$ generated by $a \in \operatorname{Aut}(G)$, several simple sufficient conditions for the LIC are known. For example, if the underlying group $G$ possesses a compact open subgroup, the dilation group can be assumed to be expanding in the sense of [2,3]. For systems on such groups, the LIC possesses a simple characterization [38]. For a general locally compact abelian group $G$, it is shown in 38 that the LIC for wavelet systems associated to $\mathcal{A}=\langle a\rangle$ is equivalent to locally integrability of the Calderón sum $t_{0}$, provided that the adjoint automorphisms are expansive in the sense of [38, Proposition 4.9]. See also [6, Proposition 2.7] for the same result on $G=\mathbb{R}^{d}$. In this latter setting, the characterization of the LIC holds in fact for any wavelet system satisfying the so-called lattice counting estimate. In [7], Bownik and the first named author show that the lattice counting estimate holds for all dilations $A \in \mathrm{GL}_{d}(\mathbb{R})$ with $|\operatorname{det} A| \neq 1$ and for almost every translation lattice $\Gamma$ with respect an invariant probability measure on the set of lattices. As a consequence, Theorem 3.2 and 3.3 are applicable for almost all wavelet systems in $L^{2}\left(\mathbb{R}^{d}\right)$ in the probabilistic sense of [7].

The remainder of this subsection is devoted to two examples for which phase cancellations in (4.5) can occur and for which such cancellations cannot be expected. Both examples take place in $L^{2}\left(\mathbb{R}^{d}\right)$. In this setting, any automorphism is given by $x \mapsto A x$ for some $A \in \mathrm{GL}_{d}(\mathbb{R})$. For such an automorphism, the modulus reads $|\operatorname{det} A|$ and the adjoint is $A^{T}$. A discrete, cocompact subgroup $\Gamma \subseteq \mathbb{R}^{d}$ is a full-rank lattice in $\mathbb{R}^{d}$, i.e., $\Gamma=C \mathbb{Z}^{d}$ for some $C \in \mathrm{GL}_{d}(\mathbb{R})$. The annihilator $\Gamma^{\perp}$ of a full-rank lattice $\Gamma \subseteq \mathbb{R}^{d}$ can be identified with the dual lattice $\Gamma^{*}=C^{\sharp} \mathbb{Z}^{d}$, where $C^{\sharp}:=\left(C^{T}\right)^{-1}$.

Example 4.1. Let $A \in \mathrm{GL}_{d}(\mathbb{R})$, let $B:=A^{T}$ and let $\Gamma=C \mathbb{Z}^{d}$ be a full-rank lattice in $\mathbb{R}^{d}$ satisfying $\Gamma^{*} \cap B^{j} \Gamma^{*}=\{0\}$ for all $j \in \mathbb{Z} \backslash\{0\}$. Examples of such pairs $(A, \Gamma)$ are $B=\beta I$ with $I$ denoting the identity matrix, $\Gamma=\mathbb{Z}^{d}$, and $\beta \in \mathbb{R}$ being such that $\beta^{j} \notin \mathbb{Q}$ for all $j \in \mathbb{Z} \backslash\{0\}$. Now, since $B^{j} \Gamma^{*}, j \in \mathbb{Z}$, are disjoint outside the origin, it follows that the set $\kappa(\alpha)$ is a singleton for each $\alpha \in \bigcup_{j \in \mathbb{Z}} B^{j} \Gamma^{*} \backslash\{0\}$. Therefore, the remainder function $R: \mathbb{R}^{d} \rightarrow[0, \infty]$ takes the form

$$
R(\omega)=\frac{1}{|\operatorname{det} C|} \sum_{\alpha \in \bigcup_{j \in \mathbb{Z}} B^{j} \Gamma^{*} \backslash\{0\}}\left|\sum_{\ell \in L} \hat{\psi}_{\ell}\left(B^{-j} \omega\right) \overline{\hat{\psi}_{\ell}\left(B^{-j}(\omega+\alpha)\right)}\right|
$$




$$
=\frac{1}{|\operatorname{det} C|} \sum_{j \in \mathbb{Z}} \sum_{k \in \Gamma^{*} \backslash\{0\}}\left|\sum_{\ell \in L} \hat{\psi}_{\ell}\left(B^{-j} \omega\right) \overline{\hat{\psi}_{\ell}\left(B^{-j} \omega+k\right)}\right| .
$$

Consequently, phase cancellation between scales cannot occur in the estimates in Theorem 4.1 , This observation fits precisely with a result by Laugesen [42]. In [42], it is proved that for wavelet systems in $L^{2}(\mathbb{R})$ with transcendental dilations $a>0$ and integer translates, which in particular implies that $\bigcap_{j \in \mathbb{Z}} a^{j} \mathbb{Z}=\{0\}$, no cancellations between scales can happen for any kind of frame bound estimate based on $w_{f}(x)$. Note that despite the fact that no phase cancellations can happen, the estimate is still optimal for tight frames. This phenomenon is due to the fact that the characterizing equations for tight wavelet systems with expansive dilation $A$ satisfying $\bigcap_{j \in \mathbb{Z}} B^{j} \Gamma^{*}=\{0\}$ are very restrictive on properties of $\psi_{\ell}$. For example, Riesz bases possessing this property have to be combined MSF wavelets [5, 10, 18].

In the previous example it was assumed that the lattices $B^{j} \Gamma^{*}, j \in \mathbb{Z}$, are disjoint outside the origin. The next example assumes that the involved lattices are nested.

Example 4.2. Let $A \in \mathrm{GL}_{d}(\mathbb{R})$, let $B:=A^{T}$ and let $\Gamma=C \mathbb{Z}^{d}$ be a full-rank lattice in $\mathbb{R}^{d}$ satisfying $B \Gamma^{*} \subset \Gamma^{*}$. In case $\Gamma=\mathbb{Z}^{d}$, this assumption is equivalent with $A$ being integer-valued. The union $\bigcup_{j \in \mathbb{Z}} B^{j} \Gamma^{*} \backslash\{0\}$ can be re-written as the disjoint union $\bigcup_{m \in \mathbb{Z}} B^{m}\left(\Gamma^{*} \backslash B \Gamma^{*}\right)$. For $\alpha=B^{m} q$, where $m \in \mathbb{Z}$ and $q \in \Gamma^{*} \backslash B \Gamma^{*}$, we have that $\kappa(\alpha)=\{j \in \mathbb{Z}: j \leq m\}$. Therefore, the remainder function $R: \mathbb{R}^{d} \rightarrow[0, \infty]$ takes the form

$$
\begin{aligned}
R(\omega) & =\frac{1}{|\operatorname{det} C|} \sum_{m \in \mathbb{Z}} \sum_{q \in \Gamma^{*} \backslash B \Gamma^{*}}\left|\sum_{j=-\infty}^{m} \sum_{\ell \in L} \hat{\psi}_{\ell}\left(B^{-j} \omega\right) \overline{\hat{\psi}_{\ell}\left(B^{-j}\left(\omega+B^{m} q\right)\right)}\right| \\
& =\frac{1}{|\operatorname{det} C|} \sum_{m \in \mathbb{Z}} \sum_{q \in \Gamma^{*} \backslash B \Gamma^{*}}\left|\sum_{n=0}^{\infty} \sum_{\ell \in L} \hat{\psi}_{\ell}\left(B^{n+m} \omega\right) \overline{\hat{\psi}_{\ell}\left(B^{n}\left(B^{m} \omega+q\right)\right)}\right| .
\end{aligned}
$$

Since the functions $t_{0}$ and $R$ are $B$-dilation periodic, i.e., $t_{0}(B \omega)=t_{0}(\omega)$ and $R(B \omega)=R(\omega)$ for a.e. $\omega \in \mathbb{R}^{d}$, the estimates (4.7) and (4.8) read

$$
b_{1}=\operatorname{esssup}_{\omega \in B(\Omega) \backslash \Omega}\left(\frac{1}{|\operatorname{det} C|} \sum_{\ell \in L} \sum_{j \in \mathbb{Z}}\left|\hat{\psi}_{\ell}\left(B^{j} \omega\right)\right|^{2}+R(\omega)\right)
$$

and

$$
a_{1}=\underset{\omega \in B(\Omega) \backslash \Omega}{\operatorname{essinf}}\left(\frac{1}{|\operatorname{det} C|} \sum_{\ell \in L} \sum_{j \in \mathbb{Z}}\left|\hat{\psi}_{\ell}\left(B^{j} \omega\right)\right|^{2}-R(\omega)\right),
$$

where $\Omega:=B(0,1)$ is the unit ball in $\mathbb{R}^{d}$, and $R: \mathbb{R}^{n} \rightarrow[0, \infty]$ is given as in (4.9). For univariate wavelets with $A=B=2$ and $\Gamma=c \mathbb{Z}, c>0$, these estimates coincidl 3 with Tchamitchian's estimates as communicated by Daubechies [19, 20]

To show that the frame bound estimates from Theorem 4.1 improve the sufficient condition based on the remainder function (4.6), note that (4.6) in the special case considered in this example simply reads

$$
\tilde{R}(\omega)=\frac{1}{|\operatorname{det} C|} \sum_{j \in \mathbb{Z}} \sum_{\alpha \in \Gamma^{*} \backslash\{0\}} \sum_{\ell \in L}\left|\hat{\psi}_{\ell}\left(B^{j} \omega\right) \overline{\hat{\psi}_{\ell}\left(B^{j} \omega+\alpha\right)}\right| .
$$

\footnotetext{
${ }^{3}$ The frame bound estimates (4.10) and (4.11) are slightly improved versions of the estimates that occur in [19, Theorem 2.9]. The improvement boils in essence down to a change of variable and taking suprema and infima differently than in the original proof.
} 
Now to see that $R(\omega) \leq \tilde{R}(\omega)$ for a.e. $\omega \in \mathbb{R}^{n}$, one simply uses the triangle inequality and notes that there is a bijection between the indices $(m, n, q) \in\left(\mathbb{Z}, \mathbb{N}, \Gamma^{*} \backslash B \Gamma^{*}\right)$ and the indices $(j, \alpha) \in \mathbb{Z} \times \Gamma^{*} \backslash\{0\}$ given by

$$
(m, n, q) \mapsto(j, \alpha), \quad \text { where } \alpha=B^{n} q \text { and } j=n+m .
$$

For $\Gamma=\mathbb{Z}^{d}$, the above two examples show the two extremes on the possible phase cancellations of Theorem 3.3 that happen for integer dilations and certain irrational dilations. For a rational dilation matrix $A \in \mathrm{GL}_{d}(\mathbb{Q})$, frame bound estimates with phase cancellations in (4.5) over infinitely many scales are clearly also possible. In fact, Laugesen remarked already in 42 that this would be possible for rational dilation in dimension one, such dilations necessarily being expansive. Recall that the analysis in the present paper does not require that the dilation is expansive, only that the 1-UCP is satisfied.

\subsection{Composite wavelets and shearlet systems}

Consider the Cartesian product $I \times J$ for two countable index sets $I$ and $J$. Let $A_{i}, B_{j} \in$ $\mathrm{GL}_{d}(\mathbb{R})$ for $i \in I$ and $j \in J$. Let $\Gamma=C \mathbb{Z}^{d}$ be a full-rank lattice in $\mathbb{R}^{d}$. The wavelet system associated with the pair $\left(\left\{A_{i} B_{j}\right\}_{(i, j) \in I \times J}, \Gamma\right)$ is a collection of functions of the form

$$
\left\{D_{A_{i} B_{j}} T_{\gamma} \psi_{\ell}\right\}_{i \in I, j \in J, \gamma \in \Gamma, \ell \in L}
$$

and forms a so-called wavelet system with composite dilations in $L^{2}\left(\mathbb{R}^{d}\right)$, see e.g., [26]. One usually assumes that one of the two family of matrices, say $\left\{A_{i}\right\}_{i \in I}$, is volume preserving. We will assume that $A_{i}^{T}, i \in I$, acts invariant on $\Gamma^{*}$, that is, $A_{i}^{T} \Gamma^{*}=\Gamma^{*}$, e.g., in case $\Gamma=\mathbb{Z}^{d}$, this assumption reads $A_{i} \in \mathrm{SL}_{d}(\mathbb{Z})$. Therefore, $\Gamma_{(i, j)}^{\perp}=B_{j}^{T} A_{i}^{T} \Gamma^{*}=B_{j}^{T} \Gamma^{*}$ for $(i, j) \in I \times J$. Thus, for composite wavelet systems satisfying the 1-UCP, an application of Theorem 4.1 yields the frame bound estimates (4.7) and (4.8), where

$$
t_{0}(\omega)=\frac{1}{|\operatorname{det} C|} \sum_{i \in I} \sum_{j \in J} \sum_{\ell \in L}\left|\hat{\psi}_{\ell}\left(A_{i}^{\sharp} B_{j}^{\sharp} \omega\right)\right|^{2}
$$

and

$$
R(\omega)=\frac{1}{|\operatorname{det} C|} \sum_{\alpha \in \bigcup_{j \in J} B_{j}^{T} \Gamma^{*} \backslash\{0\}}\left|\sum_{\ell \in L} \sum_{i \in I} \sum_{j \in \kappa(\alpha)} \hat{\psi}_{\ell}\left(A_{i}^{\sharp} B_{j}^{\sharp} \omega\right) \overline{\hat{\psi}_{\ell}\left(A_{i}^{\sharp} B_{j}^{\sharp}(\omega+\alpha)\right)}\right|
$$

with $\kappa(\alpha):=\left\{j \in J \mid \alpha \in B_{j}^{T} \Gamma^{*} \backslash\{0\}\right\}$.

The classical shearlet system is a special case of wavelets with composite dilations. For simplicity we restrict our attention to $L^{2}\left(\mathbb{R}^{2}\right)$, but we refer to [26, section 3.4] for a discussion of shearlet systems in $L^{2}\left(\mathbb{R}^{d}\right)$. One defines

$$
A=\left(\begin{array}{ll}
4 & 0 \\
0 & 2
\end{array}\right) \text { and } S=\left(\begin{array}{ll}
1 & 1 \\
0 & 1
\end{array}\right)
$$

and considers the wavelet system associated with the pair $\left(\left\{S^{k} A^{j}\right\}_{j, k \in \mathbb{Z}}, \Gamma\right)$, where $\Gamma=C \mathbb{Z}^{2}$ for some $C \in \mathrm{GL}_{d}(\mathbb{R})$. For the classical shearlet system of the form $\left\{D_{S^{k} A^{j}} T_{\gamma} \psi_{\ell}\right\}_{j, k \in \mathbb{Z}, \gamma \in \Gamma, \ell \in L}$ we find as above that the corresponding functions $t_{0}: \mathbb{R}^{2} \rightarrow \mathbb{C}$ and $R: \mathbb{R}^{2} \rightarrow[0, \infty]$ are formally given as

$$
t_{0}(\omega)=\frac{1}{|\operatorname{det} C|} \sum_{j \in \mathbb{Z}} \sum_{k \in \mathbb{Z}} \sum_{\ell \in L}\left|\hat{\psi}_{\ell}\left(\left(S^{\sharp}\right)^{k} A^{-j} \omega\right)\right|^{2}
$$


and

$$
R(\omega)=\frac{1}{|\operatorname{det} C|} \sum_{m \in \mathbb{Z}} \sum_{q \in \Gamma^{*} \backslash A \Gamma^{*}}\left|\sum_{n=0}^{\infty} \sum_{k \in \mathbb{Z}} \sum_{\ell \in L} \hat{\psi}_{\ell}\left(\left(S^{\sharp}\right)^{k} A^{n+m} \omega\right) \overline{\hat{\psi}_{\ell}\left(\left(S^{\sharp}\right)^{k} A^{n}\left(A^{m} \omega+q\right)\right)}\right| .
$$

Since any shearlet system that satisfies the CC-condition satisfies the $\alpha$-LIC, an application of Theorem 3.3 yields the following result.

Theorem 4.2. Let $L$ be a countable index set, let $\left\{\psi_{\ell}\right\}_{\ell \in L} \subset L^{2}\left(\mathbb{R}^{2}\right)$ and let $\Gamma \subset \mathbb{R}^{2}$ be a full-rank lattice. Suppose the shearlet system $\left\{D_{S^{k} A^{j}} T_{\gamma} \psi_{\ell}\right\}_{j, k \in \mathbb{Z}, \gamma \in \Gamma, \ell \in L}$ satisfies

$$
b_{1}:=\operatorname{esssup}_{\omega \in \mathbb{R}^{2}}\left(t_{0}(\omega)+R(\omega)\right)<\infty
$$

and

$$
a_{1}:=\underset{\omega \in \mathbb{R}^{2}}{\operatorname{essinf}}\left(t_{0}(\omega)-R(\omega)\right)>0,
$$

where $t_{0}$ and $R$ are given in (4.12) and (4.13), respectively. Then the shearlet system $\left\{D_{S^{k}} A^{j} T_{\gamma} \psi_{\ell}\right\}_{j, k \in \mathbb{Z}, \gamma \in \Gamma, \ell \in L}$ forms a frame for $L^{2}\left(\mathbb{R}^{2}\right)$ with bounds $a_{1}$ and $b_{1}$.

The estimates in Theorem 4.2 should be compared with previously used sufficient conditions for shearlet systems that are based on the absolute CC-condition and that do not allow for phase cancellations [39.

The rest of this subsection is devoted to cone-adapted shearlet systems. Such shearlets play a more important role in applications than the classical shearlets as they treat directions in an almost uniform manner. The cone-adapted shearlet system is a finite union of shift-invariant systems and wavelet systems with composite dilations. To introduce these systems, we define $A_{1}=A, S_{1}=S$,

$$
A_{2}=\left(\begin{array}{ll}
2 & 0 \\
0 & 4
\end{array}\right) \quad \text { and } \quad S_{2}=\left(\begin{array}{ll}
1 & 0 \\
1 & 1
\end{array}\right)
$$

For generators $\phi, \psi_{i} \in L^{2}\left(\mathbb{R}^{2}\right), i=1,2$, and full-rank lattices $\Gamma_{i}=C_{i} \mathbb{Z}^{2}, i=0,1,2$, the cone-adapted shearlet system is given as:

$$
\left\{T_{\gamma} \phi\right\}_{\gamma \in \Gamma_{0}} \cup\left\{D_{S_{i}^{k} A_{i}^{j}} T_{\gamma} \psi_{i}\right\}_{j \in \mathbb{N}_{0}, k \in\left\{-K_{j}, \ldots, K_{j}\right\}, \gamma \in \Gamma_{i}, i \in\{1,2\}}
$$

where $K_{j} \in \mathbb{N}_{0}$ for $j \in \mathbb{N}_{0}$, usually one takes $K_{j}=2^{j}$ or $K_{j}=2^{j} \pm 1$.

For brevity we assume $\Gamma_{i}=\Gamma=C \mathbb{Z}^{2}$ for $i=0,1,2$ for some $C \in \mathrm{GL}_{d}(\mathbb{R})$ so that $C^{T} A_{i} C^{\sharp}$ is integer valued for $i \in\{1,2\}$. The auto-correlation functions $t_{\alpha}: \mathbb{R}^{2} \rightarrow \mathbb{C}, \alpha \in \Gamma^{*}$, are then formally given as:

$$
\begin{aligned}
& t_{0}(\omega)=|\hat{\phi}(\omega)|^{2}+\sum_{i \in\{1,2\}} \sum_{j=0}^{\infty} \sum_{k=-K_{j}}^{K_{j}}\left|\hat{\psi}_{i}\left(\left(S_{i}^{\sharp}\right)^{k} A_{i}^{-j} \omega\right)\right|^{2}, \\
& t_{\alpha}(\omega)=\hat{\phi}(\omega) \overline{\hat{\phi}(\omega+\alpha)}+\sum_{i \in\{1,2\}} \sum_{j=0}^{m_{i}} \sum_{k=-K_{j}}^{K_{j}} \hat{\psi}_{i}\left(\left(S_{i}^{\sharp}\right)^{k} A_{i}^{-j} \omega\right) \overline{\hat{\psi}_{i}\left(\left(S_{i}^{\sharp}\right)^{k} A_{i}^{-j}(\omega+\alpha)\right)},
\end{aligned}
$$

where $\alpha \in \Gamma^{*} \backslash\{0\}$, for each $i \in\{1,2\}$, is written as $A_{i}^{m_{i}} q_{i}$ for unique $m_{i} \geq 0$ and $q_{i} \in \Gamma^{*} \backslash A_{i} \Gamma^{*}$. From the auto-correlation functions (4.15) we see that for the cases $\alpha \in C^{\sharp} \mathbb{Z}^{2} \backslash 2 C^{\sharp} \mathbb{Z}^{2}$ and 
$\alpha \in C^{\sharp}\left(4 \mathbb{Z}^{2}+(2,2)\right)$, the least amount of cancellation is possible. In this case the autocorrelation function reads

$$
t_{\alpha}(\omega)=\hat{\phi}(\omega) \overline{\hat{\phi}(\omega+\alpha)}+\sum_{i \in\{1,2\}} \sum_{k=-K_{0}}^{K_{0}} \hat{\psi}_{i}\left(\left(S_{i}^{\sharp}\right)^{k} \omega\right) \overline{\hat{\psi}_{i}\left(\left(S_{i}^{\sharp}\right)^{k}(\omega+\alpha)\right)},
$$

hence only cancellation within the 0th scale is possible. On the other hand, when $\alpha \in 4^{p} C^{\sharp} \mathbb{Z}^{2}$ for some $p \in \mathbb{N}$, then cancellations can happen within all shears and all scales $j=0, \ldots, p$ for both shearlet generators $\psi_{1}$ and $\psi_{2}$, that is, $m_{1}=m_{2}=p$ in (4.15).

As local integrability conditions can be ignored for shearlet systems, we arrive at the following Tchamitchian-type estimate for cone-adapted shearlet systems.

Theorem 4.3. Let $\phi, \psi_{i} \in L^{2}\left(\mathbb{R}^{2}\right), i=1,2$, and let $\Gamma$ be a full-rank lattice in $\mathbb{R}^{2}$. If

$$
b_{1}:=\operatorname{essup}_{\omega \in \mathbb{R}^{2}} \sum_{\alpha \in \Gamma^{*}}\left|t_{\alpha}(\omega)\right|<\infty
$$

and

$$
a_{1}:=\underset{\omega \in \mathbb{R}^{2}}{\operatorname{essinf}}\left(t_{0}(\omega)-\sum_{\alpha \in \Gamma^{*} \backslash\{0\}}\left|t_{\alpha}(\omega)\right|\right)>0,
$$

where $t_{\alpha}$ is given by (4.14) and (4.15), then the cone-adapted shearlet system

$$
\left\{T_{\gamma} \phi\right\}_{\gamma \in \Gamma} \cup\left\{D_{S_{i}^{k} A_{i}^{j}} T_{\gamma} \psi_{i}\right\}_{j \in \mathbb{N}_{0}, k \in\left\{-K_{j}, \ldots, K_{j}\right\}, \gamma \in \Gamma, i \in\{1,2\}} .
$$

is a frame for $L^{2}\left(\mathbb{R}^{2}\right)$ with bounds $a_{1}$ and $b_{1}$.

The estimates in Theorem 4.3 are improvements of the sufficient conditions for coneadapted shearlet systems as given in [37, which are based on the absolute CC-condition and do not allow for phase cancellations. Here, it should be noted that the conditions in [37] are currently the only known method for constructing cone-adapted shearlet frames with compactly supported generators. Moreover, the estimates without phase cancellation in [37] are used to "optimize" the choice of shearlet and translation lattice. It would be beneficial to instead use the improved estimates (4.16) and (4.17) for optimizing the construction of compactly supported shearlets.

\subsection{Continuous translation-invariant systems}

This section considers "continuous" translation-invariant systems with translation along the whole group, e.g., $J$ being a singleton and $\Gamma=G$. Since $G^{\perp}=\{0\}$, there is only one correlation function $t_{0}: \widehat{G} \rightarrow \mathbb{C}$, and since $J$ is a singleton, the $\infty$-UCP trivially holds. Therefore, by combining the necessary condition $A \leq t_{0} \leq B$ from [24] and Theorem 3.3, we immediately recover the following characterization of the frame property [30, 31, 50].

Corollary 4.4. Let $0<A \leq B<\infty$ and let $\left\{T_{\gamma} g_{p}\right\}_{\gamma \in G, p \in P}$ be a generalized translation-invariant system satisfying the standing hypotheses (II) -(III). The system $\left\{T_{\gamma} g_{p}\right\}_{\gamma \in G, p \in P}$ forms a frame for $L^{2}(G)$ with frame bounds $A$ and $B$ if, and only if,

$$
A \leq \int_{P}\left|\hat{g}_{p}(\omega)\right|^{2} d \mu_{P}(p) \leq B
$$

for $\mu_{\widehat{G}^{-}}$a.e. $\omega \in \widehat{G}$. 
For continuous translation-invariant systems, being a frame is equivalent to the transform $C: L^{2}(G) \rightarrow L^{2}(P \times G), f \mapsto\left\{\left\langle f, T_{\gamma} g_{p}\right\rangle\right\}_{\gamma \in G, p \in P}$ being an injective, bounded linear operator with closed range. Classical examples of such transforms are the continuous wavelet transform and the windowed Fourier transform. However, the continuous bendlet transform or, more generally, the $\ell$-th order $\alpha$-shearlet transform, recently introduced in [43], are also examples of translation-invariant transforms. For these higher-order shearlet transforms the representation-theoretic approach, utilizing orthogonality relations for irreducible, square-integrable representations of an associated locally compact group, is not directly applicable [43, Section 5]. Since no characterizations of the frame property of the higher-order $\alpha$-shearlet transform are known, we outline in the next example how such a characterization can be obtained from Corollary 4.4.

Example 4.3. Let $G=\mathbb{R}^{2}$. Define the $\alpha$-scaling operator $A_{a}: \mathbb{R}^{2} \rightarrow \mathbb{R}^{2}$ by $A_{a}\left(x_{1}, x_{2}\right)=$ $\left(a x_{1}, a^{\alpha} x_{2}\right)$ for $\alpha \in[0,1]$ and $a>0$, and define the $\ell$-th order (non-linear) shearing operator $S_{r}: \mathbb{R}^{2} \rightarrow \mathbb{R}^{2}$ by $S_{r}\left(x_{1}, x_{2}\right)=\left(x_{1}+\sum_{m=1}^{\ell} r_{m} x_{2}^{m}, x_{2}\right)$ for $r=\left(r_{1}, \ldots, r_{\ell}\right) \in \mathbb{R}^{\ell}$. The Jacobian determinants of $A_{a}$ and $S_{r}$ are $a^{1+\alpha}$ and 1 , while the inverses are $A_{a^{-1}}$ and $S_{-r}$, respectively.

Let $P=\mathbb{R}_{>0} \times \mathbb{R}^{\ell}$ and set $g_{p}=a^{-(1+\alpha) / 2} \psi\left(A_{a^{-1}} S_{-r} \cdot\right)$ for some $\psi \in L^{2}\left(\mathbb{R}^{2}\right)$ and $p=(a, r) \in$ $P$. The continuous $\ell$-th order $\alpha$-shearlet transform is simply the system $\left\{T_{\gamma} g_{p}\right\}_{\gamma \in G, p \in P}$, which reads

$$
\left\{a^{-(1+\alpha) / 2} \psi\left(A_{a^{-1}} S_{-r}(\cdot-\gamma)\right)\right\}_{a \in \mathbb{R}_{>0}, r \in \mathbb{R}^{\ell}, \gamma \in \mathbb{R}^{2}} .
$$

By Corollary 4.4, the system forms a frame with bounds $A$ and $B$ if, and only if,

$$
A \leq \int_{0}^{\infty} \int_{\mathbb{R}^{\ell}} a^{-(1+\alpha)}\left|\psi\left(A_{a^{-1}} S_{-r}(\cdot)\right)^{\wedge}(\omega)\right|^{2} d r d a \leq B \quad \text { for a.e. } \omega \in \mathbb{R}^{2} .
$$

Here, we have not specified the measure $d r d a$ on $P$; a canonical choice is $a^{-\ell-2+\alpha(\ell-1)}$ times the Lebesgue measure on $\mathbb{R}_{>0} \times \mathbb{R}^{\ell}$, but the characterization is valid for any measure on $P$ satisfying the standing hypotheses (II)-(III).

The cone-adapted version is obtained by equipping $\{(a, r): a \in(0,1], r \in R\}$ with a measure $d r d a$ (satisfying the standing hypotheses), where $R$ is a subset of $\mathbb{R}^{\ell}$; a canonical choice being $R=\left[-1-a^{1-\alpha}, 1+a^{1-\alpha}\right] \times \mathbb{R}^{\ell-1}$. Let $Q: \mathbb{R}^{2} \rightarrow \mathbb{R}^{2}$ be the permutation defined by $Q\left(x_{1}, x_{2}\right)=\left(x_{2}, x_{1}\right)$, let $\tilde{A}_{a}=Q \circ A_{a} \circ Q$, and $\tilde{S}_{r}=Q \circ S_{r} \circ Q$. The cone-adapted continuous $\ell$-th order $\alpha$-shearlet system generated by $\phi, \psi, \tilde{\psi} \in L^{2}\left(\mathbb{R}^{2}\right)$ is given by

$$
\begin{aligned}
\{\phi(\cdot-\gamma)\}_{\gamma \in \mathbb{R}^{2}} \cup\left\{a^{-(1+\alpha) / 2} \psi\left(A_{a^{-1}} S_{-r}(\cdot-\gamma)\right)\right\}_{a \in(0,1], r \in R, \gamma \in \mathbb{R}^{2}} \\
\cup\left\{a^{-(1+\alpha) / 2} \tilde{\psi}\left(\tilde{A}_{a^{-1}} \tilde{S}_{-r}(\cdot-\gamma)\right)\right\}_{a \in(0,1], r \in R, \gamma \in \mathbb{R}^{2}},
\end{aligned}
$$

and forms a frame for $L^{2}\left(\mathbb{R}^{2}\right)$ with bounds $A$ and $B$ if, and only if,

$$
\begin{aligned}
A \leq|\hat{\phi}(\omega)|^{2} & +\int_{0}^{1} \int_{R} a^{-(1+\alpha)}\left|\psi\left(A_{a^{-1}} S_{-r}(\cdot)\right)^{\wedge}(\omega)\right|^{2} d r d a \\
& +\int_{0}^{1} \int_{R} a^{-(1+\alpha)}\left|\tilde{\psi}\left(\tilde{A}_{a^{-1}} \tilde{S}_{-r}(\cdot)\right)^{\wedge}(\omega)\right|^{2} d r d a \leq B
\end{aligned}
$$

for a.e. $\omega \in \mathbb{R}^{2}$.

Acknowledgments. The authors would like to thank the referees for comments improving the presentation of the paper. The second named author gratefully acknowledges support from the Austrian Science Fund (FWF): P29462-N35. 


\section{References}

[1] D. Barbieri, E. Hernández, and A. Mayeli. Calderón-type inequalities for affine frames. Appl. Comput. Harmon. Anal., To Appear.

[2] J. J. Benedetto and R. L. Benedetto. A wavelet theory for local fields and related groups. J. Geom. Anal., 14(3):423-456, 2004.

[3] R. L. Benedetto. Examples of wavelets for local fields. In Wavelets, frames and operator theory, volume 345 of Contemp. Math., pages 27-47. Amer. Math. Soc., Providence, RI, 2004.

[4] M. Bownik. The structure of shift-invariant subspaces of $L^{2}\left(\mathbf{R}^{n}\right)$. J. Funct. Anal., 177(2):282-309, 2000.

[5] M. Bownik. Combined MSF multiwavelets. J. Fourier Anal. Appl., 8(2):201-210, 2002.

[6] M. Bownik and J. Lemvig. Affine and quasi-affine frames for rational dilations. Trans. Amer. Math. Soc., 363(4):1887-1924, 2011.

[7] M. Bownik and J. Lemvig. Wavelets for non-expanding dilations and the lattice counting estimates. Int. Math. Res. Not., 2017(23):7264-7291, 2017.

[8] M. Bownik and K. A. Ross. The structure of translation-invariant spaces on locally compact abelian groups. J. Fourier Anal. Appl., 21(4):849-884, 2015.

[9] M. Bownik and Z. Rzeszotnik. The spectral function of shift-invariant spaces on general lattices. In Wavelets, frames and operator theory, volume 345 of Contemp. Math., pages 49-59. Amer. Math. Soc., Providence, RI, 2004.

[10] M. Bownik and D. Speegle. The wavelet dimension function for real dilations and dilations admitting non-MSF wavelets. In Approximation theory, X (St. Louis, MO, 2001), Innov. Appl. Math., pages 63-85. Vanderbilt Univ. Press, Nashville, TN, 2002.

[11] C. Cabrelli and V. Paternostro. Shift-invariant spaces on LCA groups. J. Funct. Anal., 258(6):2034-2059, 2010.

[12] P. G. Casazza and O. Christensen. Weyl-Heisenberg frames for subspaces of $L^{2}(\mathbb{R})$. Proc. Amer. Math. Soc., 129(1):145-154 (electronic), 2001.

[13] P. G. Casazza, O. Christensen, and A. J. E. M. Janssen. Weyl-Heisenberg frames, translation invariant systems and the Walnut representation. J. Funct. Anal., 180(1):85-147, 2001.

[14] O. Christensen. Frames, Riesz bases, and discrete Gabor/wavelet expansions. Bull. Amer. Math. Soc. (N.S.), 38(3):273-291 (electronic), 2001.

[15] C. K. Chui and X. Shi. Bessel sequences and affine frames. Applied and Computational Harmonic Analysis, 1(1):29-49, 29-49, 1993.

[16] C. K. Chui, X. Shi, and J. Stöckler. Affine frames, quasi-affine frames, and their duals. Adv. Comput. Math., 8(1):1-17, 1998.

[17] C. K. Chui and X. L. Shi. Inequalities of Littlewood-Paley type for frames and wavelets. SIAM J. Math. Anal., 24(1):263-277, 1993. 
[18] C. K. Chui and X. L. Shi. Orthonormal wavelets and tight frames with arbitrary real dilations. Appl. Comput. Harmon. Anal., 9(3):243-264, 2000.

[19] I. Daubechies. The wavelet transform, time-frequency localization and signal analysis. IEEE Trans. Inform. Theory, 39:961-1005, 1990.

[20] I. Daubechies. Ten Lectures on Wavelets. Society for Industrial and Applied Mathematics, Philadelphia, PA, USA, 1992.

[21] I. Daubechies, A. Grossmann, and Y. Meyer. Painless nonorthogonal expansions. J. Math. Phys., 27(5):1271-1283, 1986.

[22] R. E. Edwards and E. Hewitt. Pointwise limits for sequences of convolution operators. Acta Mathematica, 113(1):181-218, 1965.

[23] H. Führ. Generalized Calderón conditions and regular orbit spaces. Colloq. Math., 120(1):103-126, 2010.

[24] H. Führ and J. Lemvig. System bandwidth and the existence of generalized shift-invariant frames. J. Funct. Anal., 276(2):563-601, 2019.

[25] K. Gröchenig. Foundations of time-frequency analysis. Applied and Numerical Harmonic Analysis. Birkhäuser Boston, Inc., Boston, MA, 2001.

[26] K. Guo, D. Labate, W.-Q. Lim, G. Weiss, and E. Wilson. Wavelets with composite dilations and their MRA properties. Appl. Comput. Harmon. Anal., 20(2):202-236, 2006.

[27] C. Heil and D. Walnut. Continuous and discrete wavelet transforms. SIAM Rev., 31:628666,1989 .

[28] E. Hernández, D. Labate, and G. Weiss. A unified characterization of reproducing systems generated by a finite family. II. J. Geom. Anal., 12(4):615-662, 2002.

[29] E. Hewitt and K. A. Ross. Abstract harmonic analysis. Vol. II: Structure and analysis for compact groups. Analysis on locally compact Abelian groups. Die Grundlehren der mathematischen Wissenschaften 152. Springer-Verlag, New York, 1970.

[30] J. W. Iverson. Subspaces of $L^{2}(G)$ invariant under translation by an abelian subgroup. J. Funct. Anal., 269(3):865-913, 2015.

[31] M. S. Jakobsen and J. Lemvig. Co-compact Gabor Systems on Locally Compact Abelian Groups. J. Fourier Anal. Appl., 22(1):36-70, 2016.

[32] M. S. Jakobsen and J. Lemvig. Reproducing formulas for generalized translation invariant systems on locally compact abelian groups. Trans. Amer. Math. Soc., 368:8447-8480, 2016.

[33] A. J. E. M. Janssen. Signal analytic proofs of two basic results on lattice expansions. Appl. Comput. Harmon. Anal., 1(4):350-354, 1994.

[34] A. J. E. M. Janssen. Duality and biorthogonality for Weyl-Heisenberg frames. J. Fourier Anal. Appl., 1(4):403-436, 1995. 
[35] A. J. E. M. Janssen. The duality condition for Weyl-Heisenberg frames. In Gabor analysis and algorithms, Appl. Numer. Harmon. Anal., pages 33-84. Birkhäuser Boston, Boston, MA, 1998.

[36] Z. Jing. On the stability of wavelet and Gabor frames (Riesz bases). J. Fourier Anal. Appl., 5(1):105-125, 1999.

[37] P. Kittipoom, G. Kutyniok, and W.-Q. Lim. Construction of compactly supported shearlet frames. Constr. Approx., 35(1):21-72, 2012.

[38] G. Kutyniok and D. Labate. The theory of reproducing systems on locally compact abelian groups. Colloq. Math., 106(2):197-220, 2006.

[39] G. Kutyniok and D. Labate. Construction of regular and irregular shearlet frames. J. Wavelet Theory Appl., 1:1-10, 2007.

[40] R. S. Laugesen. Completeness of orthonormal wavelet systems for arbitrary real dilations. Appl. Comput. Harmon. Anal., 11(3):455-473, 2001.

[41] R. S. Laugesen. Translational averaging for completeness, characterization and oversampling of wavelets. Collect. Math., 53(3):211-249, 2002.

[42] R. S. Laugesen. On affine frames with transcendental dilations. Proc. Amer. Math. Soc., 135(1):211-216, 2007.

[43] C. Lessig, P. Petersen, and M. Schäfer. Bendlets: A second-order shearlet transform with bent elements. Appl. Comput. Harmon. Anal., DOI: 10.1016/j.acha.2017.06.002, to appear in print.

[44] A. Ron and Z. Shen. Frames and stable bases for shift-invariant subspaces of $L_{2}\left(\mathbf{R}^{d}\right)$. Canad. J. Math., 47(5):1051-1094, 1995.

[45] A. Ron and Z. Shen. Gramian analysis of affine bases and affine frames. Series in approximatations and decompositions, 6:375-382, 1995.

[46] A. Ron and Z. Shen. Affine systems in $L^{2}\left(\mathbb{R}^{d}\right)$, II: Dual systems. J. Fourier Anal. Appl., 3(5):617-637, 1997.

[47] A. Ron and Z. Shen. Affine systems in $L^{2}\left(\mathbb{R}^{d}\right)$ : The analysis of the analysis operator. Journal of Functional Analysis, 148(2):408-447, 1997.

[48] A. Ron and Z. Shen. Weyl-Heisenberg frames and Riesz bases in $L_{2}\left(\mathbb{R}^{d}\right)$. Duke Math. J., 89(2):237-282, 1997.

[49] A. Ron and Z. Shen. Generalized shift-invariant systems. Constr. Approx., 22(1):1-45, 2005.

[50] M. Speckbacher and P. Balazs. Reproducing pairs and the continuous nonstationary Gabor transform on LCA groups. J. Phys. A: Math. Theor., 48(39):395201, 2015.

[51] R. Tolimieri and R. S. Orr. Poisson summation, the ambiguity function, and the theory of Weyl-Heisenberg frames. J. Fourier Anal. Appl., 1(3):233-247, 1995. 